\title{
Disparity Sensitivity and Binocular Integration in Mouse Visual Cortex Areas
}

\author{
${ }^{(1)}$ Alessandro La Chioma, ${ }^{\circ}$ Tobias Bonhoeffer, and ${ }^{\circledR}$ Mark Hübener \\ Max Planck Institute of Neurobiology, Martinsried 82152, Germany
}

Binocular disparity, the difference between the two eyes' images, is a powerful cue to generate the 3D depth percept known as stereopsis. In primates, binocular disparity is processed in multiple areas of the visual cortex, with distinct contributions of higher areas to specific aspects of depth perception. Mice, too, can perceive stereoscopic depth, and neurons in primary visual cortex (V1) and higher-order, lateromedial (LM) and rostrolateral (RL) areas were found to be sensitive to binocular disparity. A detailed characterization of disparity tuning across mouse visual areas is lacking, however, and acquiring such data might help clarifying the role of higher areas for disparity processing and establishing putative functional correspondences to primate areas. We used two-photon calcium imaging in female mice to characterize the disparity tuning properties of neurons in visual areas V1, LM, and RL in response to dichoptically presented binocular gratings, as well as random dot correlograms (RDC). In all three areas, many neurons were tuned to disparity, showing strong response facilitation or suppression at optimal or null disparity, respectively, even in neurons classified as monocular by conventional ocular dominance (OD) measurements. Neurons in higher areas exhibited broader and more asymmetric disparity tuning curves compared with V1, as observed in primate visual cortex. Finally, we probed neurons' sensitivity to true stereo correspondence by comparing responses to correlated RDC (cRDC) and anticorrelated RDC (aRDC). Area LM, akin to primate ventral visual stream areas, showed higher selectivity for correlated stimuli and reduced anticorrelated responses, indicating higher-level disparity processing in LM compared with V1 and RL.

Key words: binocular disparity; calcium imaging; higher visual areas; mouse visual cortex; ocular dominance; random dot correlogram

\section{Significance Statement}

A major cue for inferring 3D depth is disparity between the two eyes' images. Investigating how binocular disparity is processed in the mouse visual system will not only help delineating the role of mouse higher areas for visual processing, but also shed light on how the mammalian brain computes stereopsis. We found that binocular integration is a prominent feature of mouse visual cortex, as many neurons are selectively and strongly modulated by binocular disparity. Comparison of responses to correlated and anticorrelated random dot correlograms (RDC) revealed that lateromedial area (LM) is more selective to correlated stimuli, while less sensitive to anticorrelated stimuli compared with primary visual cortex (V1) and rostrolateral area $(\mathrm{RL})$, suggesting higher-level disparity processing in LM, resembling primate ventral visual stream areas.

\section{Introduction}

A fundamental ability of the mammalian visual system is combining information from both eyes into a unified percept of the $3 \mathrm{D}$ world. Each eyes' retina receives an image of the environment

Received May 4, 2020; revised Sep. 18, 2020; accepted Sep. 22, 2020.

Author contributions: A.L.C. and M.H. designed research; A.L.C. performed research; A.L.C. analyzed data; A.L.C., T.B., and M.H. wrote the paper.

This work was supported by the Max Planck Society and a Boehringer Ingelheim Ph.D. fellowship (A.L.C.). We thank Pieter Goltstein for setting up intrinsic signal imaging hardware and software. We also thank Max Sperling for the development and maintenance of data acquisition software.

A. La Chioma's present address: Center for Neural Science, New York University, New York, NY 10003.

The authors declare no competing financial interests.

Correspondence should be addressed to Mark Hübener at mark@neuro.mpg.de.

https://doi.org/10.1523/JNEUROSCI.1060-20.2020

Copyright $\odot 2020$ the authors from a slightly different vantage point, such that a given object's image can fall on non-corresponding locations on the two retinae, depending on the object's distance from the observer. The accurate sensing of the difference between the two retinal images, called binocular disparity, is the first critical step underlying binocular fusion and stereoscopic depth perception (Gonzalez and Perez, 1998). In primates, many visual cortex areas play a role in this task, with distinct representations of binocular disparity among different areas (Parker, 2007; Welchman, 2016). For example, compared with the primary visual cortex (V1), neurons in extrastriate areas show broader disparity tuning and encode a wider range of disparities. Likewise, tuning curves of most V1 neurons are symmetric, but are often asymmetric in extrastriate areas (Cumming and DeAngelis, 2001; DeAngelis and Uka, 2003). Moreover, neurons in V1 and in dorsal stream areas, such 
as the middle temporal (MT) area and the medial superior temporal (MST) area, respond to both binocularly correlated and anticorrelated stimuli (Cumming and Parker, 1997; Takemura et al., 2001; Krug et al., 2004), whereas ventral stream areas, such as V4 and inferior temporal cortex, display weaker or no responses to anticorrelated stimuli, reflecting higher-level processing of disparity signals and a close correlation with the perception of stereo depth (Janssen et al., 2003; Tanabe et al., 2004). Finally, visual areas across ventral and dorsal streams show different selectivities for either near or far stimuli (Cléry et al., 2018; Nasr and Tootell, 2018). Thus, the tuning characteristics of disparity sensitive neurons in the primate visual system have helped clarifying the hierarchy of visual areas and their distinct roles for stereo-based depth processing.

The mouse has become a key model for understanding visual cortex function, largely because of its experimental tractability (Niell, 2015; Glickfeld and Olsen, 2017). As in other mammals, mouse visual cortex consists of V1 and multiple higher areas with specific interconnections and different proposed roles for visual information processing, which are still relatively poorly understood, however (Wang et al., 2011, 2012; Andermann et al., 2011; Marshel et al., 2011; Zhuang et al., 2017; Han et al., 2018; de Vries et al., 2020).

Mice can discriminate stereoscopic depth (Samonds et al., 2019) and disparity-sensitive neurons similar to those characterized in other mammals were found in V1 (Scholl et al., 2013) and in higher-order lateromedial (LM) and rostrolateral (RL) areas (La Chioma et al., 2019). Clear differences in neurons' preferred disparities were observed across these areas, with area RL being specialized for disparities corresponding to nearby visual stimuli (La Chioma et al., 2019).

Beyond these differences, binocular disparity has not been analyzed in detail in the different areas of mouse visual cortex. Obtaining this information should not only contribute to a better understanding of the role of higher areas for visual processing, but it will also facilitate establishing functional correspondences to primate areas. Here, we characterize binocular disparity in V1 and areas LM and RL, which jointly contain the largest representation of the binocular visual field across mouse visual cortex. Using two-photon calcium imaging, we determined the disparity tuning properties of neurons using dichoptically presented binocular gratings, as well as correlated and anticorrelated random dot correlograms (RDC).

We found that, across these areas, many neurons were tuned to disparity. Binocularly presented stimuli caused strong response facilitation or suppression at optimal or null disparity, respectively, even in neurons classified as monocular by conventional ocular dominance (OD) measurements. While none of the areas studied showed a large-scale spatial organization for disparity preference, nearby neurons within $10 \mu \mathrm{m}$ had similar tuning properties. Disparity tuning curves in higher areas were broader and more asymmetric compared with those in V1, as observed in primate visual cortex. Finally, a fraction of neurons across areas responded to anticorrelated RDC (aRDC), with tuning curves inverted compared with correlated RDC ( $\mathrm{CRDC}$ ). Area LM, similar to primate ventral visual stream areas, showed substantially fewer anticorrelated responses, suggesting a higher-level analysis of disparity signals in LM compared with V1 and RL.

\section{Materials and Methods}

Virus injection and cranial window implantation

All experimental procedures were conducted in accordance with the institutional guidelines of the Max Planck Society and the local government (Regierung von Oberbayern). A total of 13 female adult C57/BL6 mice were used, housed with littermates (three to four per cage) in a 12/12 h light/dark cycle in individually ventilated cages, with access to food and water ad libitum.

Cranial window implantations were performed at 10-12 weeks of age, following the procedures described in (La Chioma et al., 2019). Briefly, mice were anesthetized by intraperitoneal injection of a mixture of fentanyl $(0.075 \mathrm{mg} / \mathrm{kg})$, midazolam $(7.5 \mathrm{mg} / \mathrm{kg})$, and medetomidine $(0.75 \mathrm{mg} / \mathrm{kg})$. Virus injections were performed through a circular craniotomy (4-5 $\mathrm{mm}$ in diameter) into the right hemisphere (images in Fig. $1 C, E$ were mirrored for consistency with cited references), at three to five sites in the binocular region of $\mathrm{V} 1$ and $\sim 0.5-1 \mathrm{~mm}$ more lateral (corresponding to the location of areas LM and RL), using AAV2/1.Syn. mRuby2.GSG.P2A.GCaMP6s.WPRE.SV40 (Rose et al., 2016; Addgene viral prep \#50942-AAV1). Following injections, the craniotomy was sealed flush with the brain surface using a glass cover slip. A custom machined aluminum head-plate was attached to the skull using dental cement to allow head-fixation during imaging. Expression of the transgene was allowed for $2.5-3$ weeks before imaging.

\section{Intrinsic signal imaging}

Intrinsic signal imaging was used to localize areas V1, LM, and RL. Imaging was performed under anesthesia two to four weeks after cranial window implantation, as detailed previously (La Chioma et al., 2019).

\section{In vivo two-photon imaging}

Two-photon imaging was performed $3-17$ weeks after cranial window implantation for experiments under anesthesia (eight mice total) and 612 weeks after cranial window implantation for experiments in awake animals (five mice total). For imaging under anesthesia, mice were initially anesthetized by intraperitoneal injection of a mixture of fentanyl $(0.030 \mathrm{mg} / \mathrm{kg})$, midazolam $(3.0 \mathrm{mg} / \mathrm{kg})$, and medetomidine $(0.30 \mathrm{mg} / \mathrm{kg})$. Additional anesthetic mixture ( $25 \%$ of the induction dose) was injected subcutaneously $60 \mathrm{~min}$ after the initial injection and then every 30 $40 \mathrm{~min}$ to maintain anesthesia. Images were acquired using a custombuilt two-photon microscope (La Chioma et al., 2019) equipped with an $8-\mathrm{kHz}$ resonant galvanometer scanner, resulting in frame rates of $17.6 \mathrm{~Hz}$ at an image resolution of $750 \times 900$ pixels $(330 \times 420 \mu \mathrm{m})$. The illumination source was a Ti:Sapphire laser with a DeepSee pre-chirp unit (Spectra Physics MaiTai eHP), set to an excitation wavelength of $940 \mathrm{~nm}$. Laser power was $10-35 \mathrm{~mW}$ as measured after the objective $(16 \times, 0.8 \mathrm{NA}, \mathrm{Nikon})$. For awake imaging, the animals were head-fixed on top of an air suspended Styrofoam ball (diameter, $20 \mathrm{~cm}$ ), allowing the mouse to run during stimulus presentation and data acquisition (Dombeck et al., 2007).

\section{Monitoring eye position}

During two-photon imaging, both eyes were continuously imaged with an infrared video camera (The Imaging Source, frame rate $30 \mathrm{~Hz}$ ). Pupil position and diameter were monitored online using custom-written software (LabVIEW, National Instruments) based on Sakatani and Isa (2007). Analysis of pupil position was also performed post hoc to test whether either eye had changed position over the course of the experiment. Approximately $10 \%$ of the imaging experiments under anesthesia were discarded owing to eye drifts.

\section{Visual stimulation: dichoptic stimulation}

All visual stimuli presented during two-photon imaging in anesthetized mice (Figs. 1-6) were displayed through a haploscope, consisting of two separate mirrors and two separate display monitors to enable independent stimulation of each eye (Fig. 1D). Each mirror (silver coated, $25 \times$ $36 \times 1.05 \mathrm{~mm}$, custom-made, Thorlabs), mounted on a custom designed, 3D-printed plastic holder, was independently positioned at an angle of $\sim 30^{\circ}$ to the longitudinal axis of the mouse, contacting the snout $2-4 \mathrm{~mm}$ anterior to the medial palpebral commissure of each eye. A shield made of black paper board and tape was used to prevent stimulus cross-talk between eyes and monitors. Each mirror redirected the field of view of each eye onto a separate display monitor located on each side of the animal at a distance of $21 \mathrm{~cm}$, with an actual stimulation area subtending $65^{\circ}$ in elevation and 
A

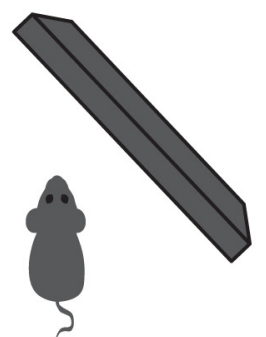

C

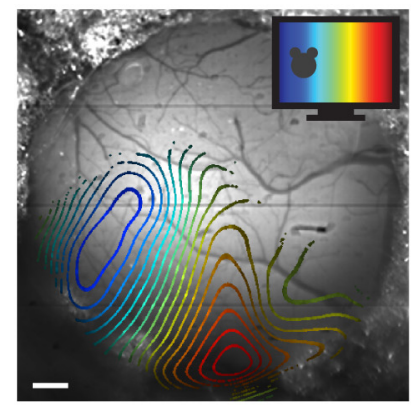

B

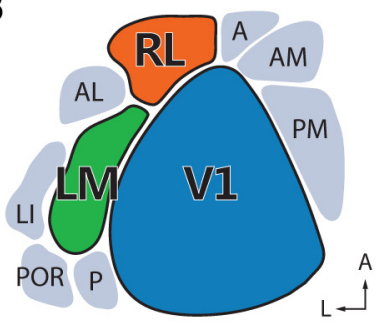

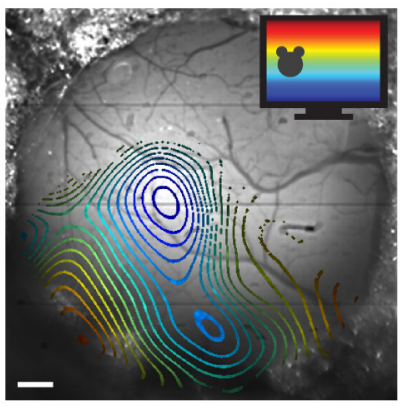
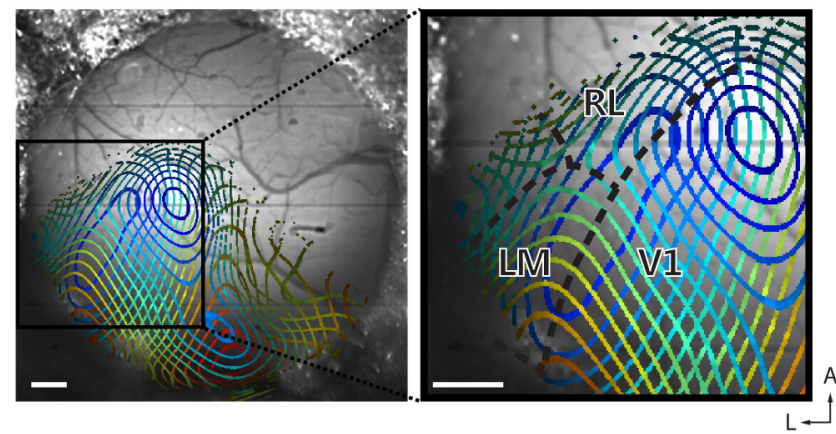

D

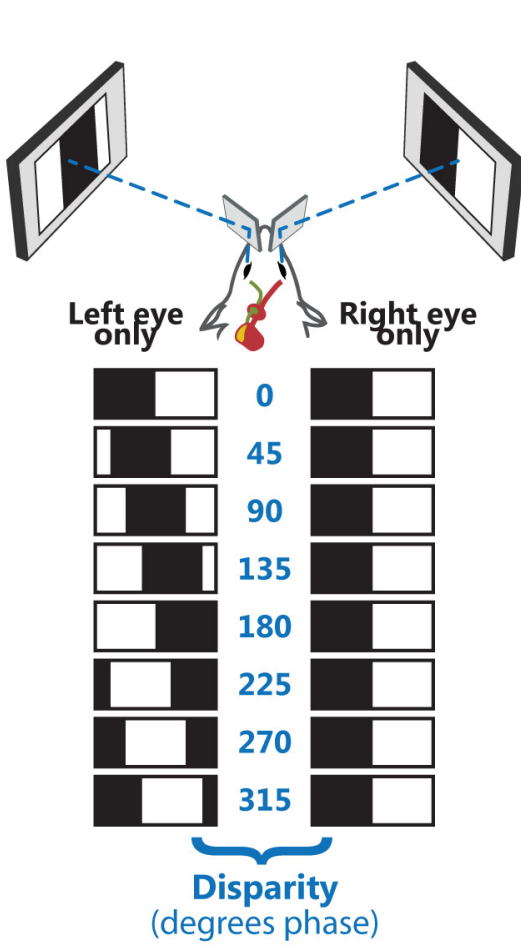

$\mathbf{E}$
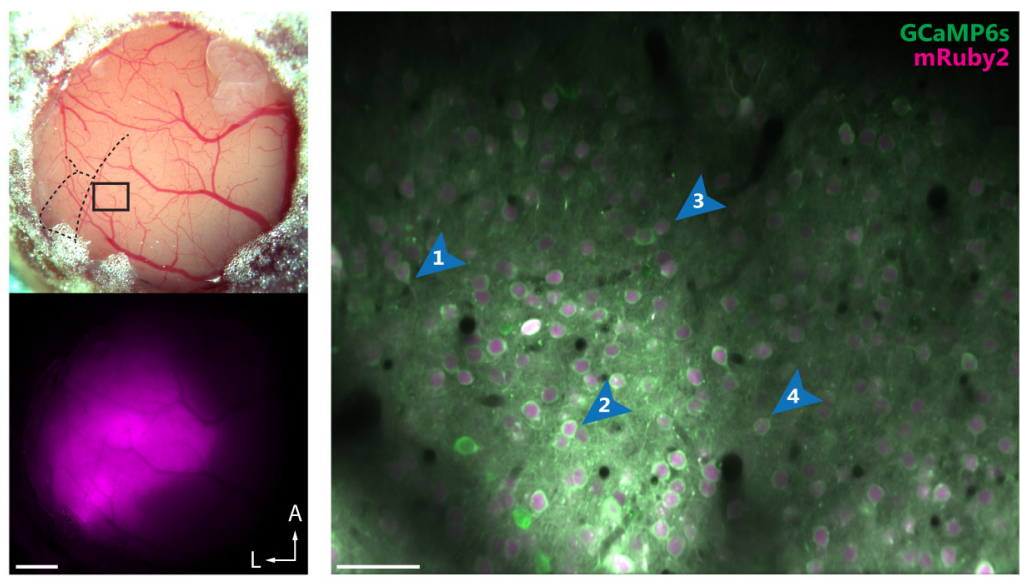

$\mathbf{F}$

Grating phase disparities

C I $0 \quad 45 \quad 90 \quad 135180225270315$

Cell 1 $\mathrm{DI}=0.74$

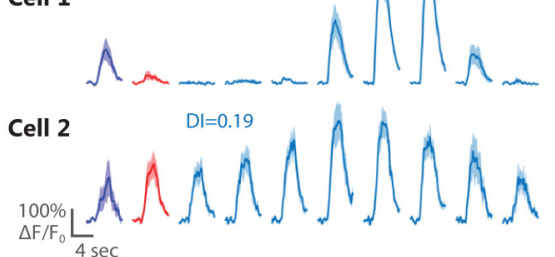

Grating phase disparities C I $\quad 0 \quad 45 \quad 90 \quad 135 \quad 180225270 \quad 315$

Cell 3

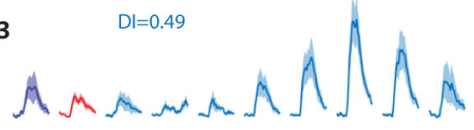
Cell $4 \quad \mathrm{DI}=0.54$

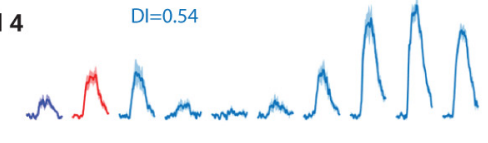

Figure 1. Identification and targeting of areas V1, LM, and RL for two-photon calcium imaging. $A$, Schematic of stimulus presentation for mapping the retinotopic organization of mouse visual cortex areas. Left, Top view. Right, Periodic bar stimulus displayed with spherical correction. $\boldsymbol{B}$, Schematic of the location of V1 and several higher-order areas of mouse visual cortex in the left hemisphere. The color code for areas V1 (blue), LM (green), and RL (orange) is used throughout the figures. C, Retinotopic maps from an example mouse. Contour plots of retinotopy are overlaid with an image of the brain surface. Contour lines depict equally spaced, iso-elevation and iso-azimuth lines as indicated by the color code. Panels from left to right, Contour plot for azimuth; contour plot for elevation; overlay of azimuth and elevation contours; enlarged view of cortical areas V1, LM, and RL. The boundaries between these areas (dashed black lines) can be reliably delineated. Scale bars: $500 \mu \mathrm{m}$. D, Schematic illustrating dichoptic grating stimulation. Top, Haploscope apparatus for dichoptic presentation of visual stimuli. Bottom, Drifting gratings are dichoptically presented at varying interocular phase disparities. Eight equally spaced interocular grating disparities $\left(0-315^{\circ}\right.$ phase) are produced by systematically varying the initial phase (position) of the grating presented to one eye relative to the phase of the grating presented to the other eye. $\boldsymbol{E}$, Two-photon imaging using the calcium indicator GCaMP6s co-expressed with the structural marker mRuby2. Top left, Image of a cranial window 6.5 weeks after implantation. Bottom left, Epifluorescence image showing the expression bolus, with fluorescence signal from mRuby2. Right, Example two-photon imaging plane acquired $\sim 180 \mu \mathrm{m}$ below the cortical surface in area V1. The image shows a mean-intensity projection (20,000 frames, shift corrected) with fluorescence signal from GCaMP6s (green) and mRuby2 (magenta). The cortical location of this imaging plane is indicated in the top left panel. Scale bars: $500 \mu \mathrm{m}$ (left panels) and $50 \mu \mathrm{m}$ (right panel). $\boldsymbol{F}$, Visually-evoked calcium transients $\left(\Delta \mathrm{F} / F_{0}\right)$ of four example neurons indicated by the blue arrowheads in $\boldsymbol{E}$. For each cell, the responses to monocular drifting gratings presented to either the contralateral (blue) or ipsilateral eye (red) are shown on the left. Responses to the eight interocular phase disparities of dichoptic gratings are shown on the right (cyan), along with the corresponding $\mathrm{DI}$. The fluorescence time courses are plotted as mean $\Delta \mathrm{F} / \mathrm{F}_{0}$ and SEM (lines and shaded areas) calculated across stimulus trials. 
$70^{\circ}$ in azimuth for each eye. The two 21-inch LCD monitors (Dell P2011Ht, gamma corrected, refresh rate of $60 \mathrm{~Hz}$, spatial resolution of $1600 \times 900$ pixels) were mounted on custom machined metal holders that allowed flexible and reproducible positioning of each monitor independently. To minimize light contamination of data images from visual stimulation, the LED backlight of the monitors were flickered at $16 \mathrm{kHz}$ such that they were synchronized to the line clock of the resonant scanner (Leinweber et al., 2014). As a result, the LED backlight was only active during the turnaround intervals of the scan phase, which were not used for image generation (mean luminance with $16-\mathrm{kHz}$ flickering: white, $5.2 \mathrm{~cd} / \mathrm{m}^{2}$; black $0.01 \mathrm{~cd} / \mathrm{m}^{2}$ ).

For dichoptic stimulation with RDC during two-photon imaging in awake mice (Figs. 7, 8), modified eye shutter glasses (3D Vision 2, Nvidia) were used (La Chioma et al., 2019). The glasses consisted of a pair of liquid crystal shutters, one for each eye, that rapidly $(60 \mathrm{~Hz})$ alternated their electro-optical state, i.e., either occluded or transparent to light. In one frame sequence, the left eye shutter was occluded while the right eye shutter was transparent, and vice versa for the next frame, with alternations synchronized to the monitor refresh rate $(120 \mathrm{~Hz})$. Synchrony between the shutter glasses and the monitor was accomplished with an infra-red wireless emitter. For optimal positioning of the eye shutters, the glasses' frame was carefully disassembled, preserving the enclosed electronics, and the two shutters were mounted on two independently adjustable arms. The display monitor (Acer GN246HL, 24 inches, $120-\mathrm{Hz}$ refresh rate, spatial resolution $1600 \times 900$ pixels) was placed in front of the mouse at a distance of $13 \mathrm{~cm}$ from the eyes (luminance measured through the transparent shutter: white, $21.6 \mathrm{~cd} / \mathrm{m}^{2}$; black $0.05 \mathrm{~cd} / \mathrm{m}^{2}$ ). To reduce light contamination of two-photon images from visual stimulation, the microscope objective was shielded using black tape. Visual stimuli were generated using custom-written code for MATLAB (MathWorks) with the Psychophysics Toolbox (Brainard, 1997; Kleiner et al., 2007). For dichoptic stimulation through the haploscope, the code was run on a Dell PC (T7300) equipped with a Nvidia Quadro K600 graphics card and using a Linux operating system to ensure better performance and timing in dual-display mode, as recommended (Kleiner, 2010). For dichoptic stimulation through eye shutter glasses, the code was run on a Dell PC (Precision T7500) equipped with a Nvidia Quadro K4000 graphics card and using Windows 10.

\section{Visual stimulation: retinotopic mapping}

When using the haploscope for dichoptic stimulation (Figs. 1-6), retinotopic mapping was performed to ensure that the field of view of each eye was roughly redirected onto the central area of its display monitor. Visual stimuli consisted of vertical and horizontal patches of drifting gratings, presented to each eye in six vertical (size $16 \times 65^{\circ}$, width $\times$ height) and five horizontal (size $70 \times 13^{\circ}$, width $\times$ height) locations (eight consecutive directions in pseudorandom sequence; $\mathrm{SF}, 0.05 \mathrm{cpd}$; TF, $2 \mathrm{~Hz}$; duration of each stimulus patch, $4 \mathrm{~s}$; interstimulus interval, $2 \mathrm{~s}$; number of stimulus trials, two to four). Retinotopic maps for each eye were generated immediately after completing stimulation (for details on analysis, see Online analysis of retinotopic mapping). If the center of the ensemble receptive field (RF) of one eye was closer than $\sim 20^{\circ}$ to the screen edge, the position of the haploscope mirror of that eye was adjusted and the retinotopic mapping was repeated.

\section{Visual stimulation: monocular drifting gratings}

When using the haploscope for dichoptic stimulation (Figs. 1-6), slight misalignment of the two mirrors could cause the artefactual rotation of one eye's field of view relative to that of the other eye. To estimate the rotation offset between each eye's field of view (see Eye rotation offset online analysis), monocular drifting gratings were used. Sinusoidal oriented gratings were presented, to each eye separately, at 12 or 16 equally spaced drifting directions $\left(30-360^{\circ}\right.$ or $\left.22.5-360^{\circ}\right)$. Gratings were displayed in full-field and at $100 \%$ contrast, at a SF of $0.05 \mathrm{cpd}$ and a TF of $2 \mathrm{~Hz}$. Each stimulus was displayed for $3 \mathrm{~s}$ (six grating cycles, randomized initial spatial phase) preceded by an interstimulus interval of $2 \mathrm{~s}$ with a blank (gray) screen with the same mean luminance as during the stimulus period. During presentation of a grating stimulus on one monitor, the other monitor displayed a blank screen. Each stimulus was repeated for two to three trials, in pseudorandomized sequence across drifting directions and eyes. In a given experiment, all subsequent stimuli presented through the haploscope were displayed by correcting for the eye rotation offset, which was estimated by online analysis of the neuronal responses, assuming matched orientation preference between the two eyes in adult mice (Wang et al., 2010; for details on analysis, see Eye rotation offset online analysis).

To measure OD as well as facilitation/suppression (Fig. 3), sinusoidal oriented gratings were presented with the following stimulus parameters: two directions of drifting vertical gratings $\left(90^{\circ}\right.$, rightward; $270^{\circ}$, leftward); one of three possible SFs, spaced by 2 octaves, among $0.01 \mathrm{cpd}$, 0.05 and $0.10 \mathrm{cpd}$; TF of $2 \mathrm{~Hz}$; stimulus interval of $2 \mathrm{~s}$; interstimulus interval of $4 \mathrm{~s}$; each stimulus was repeated for six trials, in pseudorandomized sequence across drifting directions and eyes, and interleaved with dichoptic drifting gratings as a part of the same stimulation block.

\section{Visual stimulation: dichoptic drifting gratings}

Sinusoidal, drifting vertical gratings were dichoptically presented to both eyes, at varying interocular disparities. Different interocular grating disparities were generated by varying the initial phase (position) of the grating presented to one eye relative to the phase of the grating presented to the other eye across the full grating cycle. Eight equally spaced phase disparities ( $45-360^{\circ}$ phase, spacing $45^{\circ}$ phase) were used at a SF of $0.01 \mathrm{cpd}$. For each stimulus, drift direction (leftward or rightward), TF, and SF were kept constant across eyes. The other grating stimulus parameters were the same as for the monocular drifting gratings. Gratings were presented in pseudorandomized sequence across disparities and drifting directions, with five to six trials for each stimulus condition.

\section{Visual stimulation: $R D C$}

RDC consisted of a pattern of random dots, presented to both eyes in a dichoptic fashion. Between the left and the right eye stimulus patterns, a spatial offset along the horizontal axis was introduced to generate interocular disparities. A total of 19 different RDC conditions were presented, covering a range of horizontal disparities between $-26.39^{\circ}$ and $+26.39^{\circ}$. The different RDC conditions were obtained by dividing the entire range of horizontal disparities into 19 nonoverlapping bins (bin width $2.78^{\circ}$ ) and assigning each bin to one of the 19 RDC conditions (e.g., $[-1.39+$ 1.39], [+1.39+4.17], etc.). Each RDC stimulus was generated by randomly drawing a disparity value from a given bin and displaying a random pattern of dots (all dots having the same interocular disparity) for $0.15 \mathrm{~s}$, after which a new random disparity value from the same bin was drawn and a new random pattern of dots was displayed for $0.15 \mathrm{~s}$, and so forth, up to a total duration of $4 \mathrm{~s}$ ( 27 patterns of dots). The circular dots (diameter, $12^{\circ}$ ) were displayed in full-field at random locations (with overlap possible), with an overall density of $25 \%$ (percentage of pixels occupied by dots assuming no overlap); $50 \%$ of the dots (diameter, $12^{\circ}$ ) were bright (brightness $77 \%$ ) and $50 \%$ of the dots were dark (brightness $23 \%$ ) against a gray background. For binocularly correlated RDC, the dots were displayed with the same contrast between the two eyes, whereas for aRDC, the dots were displayed with opposite contrast between the two eyes. Each RDC condition was presented for 10 stimulus trials in pseudorandomized sequence, with individual trials separated by an interstimulus interval of $2 \mathrm{~s}$. RDC were displayed applying spherical correction for stimulating in spherical visual coordinates using a flat monitor.

\section{Visual stimulation: RF elevation}

For experiments using RDC, RF elevation for individual neurons was determined using horizontal bars of drifting gratings (SF, $0.03 \mathrm{cpd}$; TF, $2 \mathrm{~Hz}$; contrast, $60 \%$; duration of each stimulus bar, $4 \mathrm{~s}$; interstimulus interval, $1 \mathrm{~s}$; number of stimulus trials, 6-10). Bar stimuli were presented binocularly and displayed at 11 different vertical locations on a gray background (adjacent locations were $7.3^{\circ}$ apart, $50 \%$ bar overlap), covering approximately from $-45^{\circ}$ to $42^{\circ}$ in elevation in total. To determine RF elevation, a $1 \mathrm{D}$ RF was obtained by fitting a cell's response with a Gaussian model as a function of the vertical stimulus positions, and the position in elevation corresponding to the RF peak was taken (only when model fit $R^{2}>0.5$ ). 


\section{Analysis of imaging data}

Imaging data were processed using custom-written MATLAB software, as detailed previously (La Chioma et al., 2019). Briefly, (1) images were aligned using a rigid motion registration; (2) regions of interest (ROIs) were selected by manually drawing circular shapes around cell somas; (3) the fluorescence time course of each cell was corrected for neuropil contamination and computed as $\mathrm{F}_{\text {cell_corrected }}=\mathrm{F}_{\text {cell_raw }}-r \times \mathrm{F}_{\text {neuropil }}$, where $\mathrm{F}_{\text {cell_raw }}$ is the raw fluorescence time course of the cell extracted by averaging all pixels within the somatic ROI, $F_{\text {neuropil }}$ was extracted from an annular neuropil ROI centered around the somatic ROI $(3-13 \mu \mathrm{m}$ from the border of the somatic ROI), and $r$ is a contamination factor set to 0.7 (Kerlin et al., 2010; Chen et al., 2013). For experiments in awake mice (Figs. 7, 8), imaging data were processed using the Suite2P toolbox in MATLAB (Pachitariu et al., 2016), which entailed image registration, segmentation of ROIs, and extraction of calcium fluorescence time courses. Relative changes in fluorescence signals $\left(\Delta \mathrm{F} / \mathrm{F}_{0}\right)$ were calculated, for each stimulus trial independently, as $\left(\mathrm{F}-\mathrm{F}_{0}\right) / \mathrm{F}_{0}$, where $\mathrm{F}_{0}$ was the average over a baseline period of $1 \mathrm{~s}$ immediately before onset of the visual stimulus.

\section{Analysis of retinotopic mapping of higher visual areas}

Retinotopic maps for azimuth and elevation were generated using the temporal phase method (Kalatsky and Stryker, 2003) on images obtained with intrinsic signal imaging, as described (La Chioma et al., 2019). The boundary between $\mathrm{V} 1$ on the medial side and areas $\mathrm{LM}, \mathrm{AL}$, and RL on the lateral side was identified by a reversal at the vertical meridian, as indicated by the longer axis of the elliptically shaped contour on the vertical meridian. The boundaries between LM and AL, and between AL and RL were identified as a reversal near the horizontal meridian (Kalatsky and Stryker, 2003; Marshel et al., 2011; Garrett et al., 2014). The binocular regions of areas V1, LM, and RL were then specifically targeted for two-photon imaging, by using the blood vessels as landmarks, which could be reliably recognized in the two-photon images.

\section{Online analysis of retinotopic mapping}

Data images of the recording were analyzed online ("on-the-fly") pixel by pixel, by calculating a $\Delta \mathrm{F} / \mathrm{F}$ for each pixel and grating patch location, averaged across trials, for each eye independently. Azimuth and elevation maps were generated for each eye by counting, for each vertical and horizontal stimulus location presented to one eye, the number of pixels that best responded to it (only pixels with an averaged $\Delta \mathrm{F} / \mathrm{F}_{0}$ above zero for any given location were considered). Given the high number of total pixels in the images $(675,000)$, analyzed regardless of individual cell's ownership, this online analysis provided a good estimate of the center of the overall RF across cells from that imaging plane, as confirmed by comparison to individual cells' RFs analyzed post hoc (data not shown).

\section{Eye rotation offset online analysis}

To estimate the eye rotation offset potentially caused by the haploscope mirrors (see Visual stimulation: monocular drifting gratings), data images containing responses to drifting gratings were analyzed online (on-thefly) pixel by pixel, by calculating $\Delta \mathrm{F} / \mathrm{F}$ for each pixel and grating direction, averaged across trials. For each eye independently, responsive and orientation-tuned pixels were selected on the basis of an orientation selectivity index (OSI), scaled by the maximum relative fluorescence change, with OSI calculated for each pixel as the normalized length of the mean response vector (Ringach et al., 2002; Mazurek et al., 2014):

$$
\text { OSI }=\left|\frac{\sum_{k} R\left(\theta_{k}\right) \exp \left(i \theta_{k}\right)}{\sum_{k} R\left(\theta_{k}\right)}\right|,
$$

where $R\left(\theta_{k}\right)$ is the mean $\Delta \mathrm{F} / \mathrm{F}$ response to the orientation angle $\theta_{k}$. The angle of the same mean response vector was taken as the preferred orientation of that pixel. For pixels with OSI $>0.3$ for both eye-specific stimuli, the difference in preferred orientation between left and right eye was calculated, and the average across all selected pixels was taken as the rotational offset between the eyes' fields of view (dO, mean \pm SD across all imaging planes $26.3 \pm 6.6^{\circ}$ ). Subsequent stimulations (dichoptic gratings) were presented by correcting stimulus orientation by $-\mathrm{dO} / 2$ and $+\mathrm{dO} / 2$ for stimuli presented to the left and right eye, respectively. Note that dichoptic gratings are referred to as vertical throughout the manuscript for simplicity, although they were not vertical in absolute terms because of the offset correction.

\section{Responsive cells}

Cells were defined visually responsive when $\Delta \mathrm{F}_{\text {peak }} / \mathrm{F}_{0}>4 \times \sigma_{\text {baseline }}$ in at least $50 \%$ of the trials of the same stimulus condition, where $\Delta \mathrm{F}_{\text {peak }}$ is the peak $\Delta \mathrm{F} / \mathrm{F}_{0}$ during the stimulus period of each trial, and $\sigma_{\text {baseline }}$ is the SD calculated across the $\mathrm{F}_{0}$ of all stimulus trials and conditions of the recording. For grating stimuli, the mean $\Delta \mathrm{F} / \mathrm{F}_{0}$ over the entire stimulus interval ( $2 \mathrm{~s}$ ) of each trial was calculated. For RDC stimuli, the mean $\Delta \mathrm{F} /$ $\mathrm{F}_{0}$ of each trial was calculated over a time window of $1 \mathrm{~s}$ centered around $\Delta \mathrm{F}_{\text {peak }}$.

\section{Disparity selectivity index (DI)}

For each cell responsive to dichoptic gratings, a DI was calculated, given by the normalized length of the mean response vector across the eight phase disparities of the drift direction that elicited the stronger activation (Scholl et al., 2013, 2015):

$$
\mathrm{DI}=\left|\frac{\sum_{k} R\left(\Phi_{k}\right) \exp \left(i \Phi_{k}\right)}{\sum_{k} R\left(\Phi_{k}\right)}\right|,
$$

where $R\left(\Phi_{k}\right)$ is the mean $\Delta \mathrm{F} / \mathrm{F}$ response to the interocular phase disparity $\Phi_{k}$. Cells were defined disparity-tuned to dichoptic gratings if $\mathrm{DI}>0.3$. Using more stringent criteria for defining disparity-tuned cells $(\mathrm{DI}>0.5)$ did not qualitatively affect the results and the statistical significance of all analyses. In addition, using more stringent criteria for defining responsive cells $\left(\Delta \mathrm{F}_{\text {peak }} / \mathrm{F}_{0}>8 \times \sigma_{\text {baseline }}\right)$ did not result in a significant change in the DI distribution for each area (data not shown), ruling out that neuronal calcium signals with low signal-to-noise ratio affected the measurement of disparity selectivity with gratings. Note that the calculation of DI is based on a circular metric. As such, DI could be computed only for responses to dichoptic gratings, but not for responses to RDC, which are not circular. Cells were defined disparity-tuned to cRDC or aRDC when at least $50 \%$ of the tuning curve variance $\left(R^{2}\right)$ could be accounted for by the Gabor model fit (see Disparity tuning curve fit). Using more stringent criteria for defining cells responsive to cRDC or aRDC $\left(\Delta \mathrm{F}_{\text {peak }} / \mathrm{F}_{0}>8 \times \sigma_{\text {baseline }}\right)$ did not qualitatively affect the results and the statistical significance of all analyses (data not shown), suggesting that the measurement of disparity sensitivity to CRDC and aRDC across areas was not affected by low signal-to-noise ratios.

\section{$O D$ index $(O D I)$}

OD was determined for responsive cells by calculating the ODI using eye-specific responses to drifting gratings:

$$
\text { ODI }=\frac{\mathrm{R}_{\text {contra }}-\mathrm{R}_{\text {ipsi }}}{\mathrm{R}_{\text {contra }}+\mathrm{R}_{\text {ipsi }}}
$$

where $\mathrm{R}_{\text {contra }}$ and $\mathrm{R}_{\mathrm{ipsi}}$ are the mean $\Delta \mathrm{F} / \mathrm{F}_{0}$ responses (across trials) to the preferred grating direction and SF presented to either the contralateral or ipsilateral eye, respectively. Contralateral and ipsilateral dominance are indicated by an ODI of 1 or -1 , respectively. A cell equally activated by either eye stimulation has an ODI $=0$.

\section{Facilitation index (FI) and suppression index (SI)}

To quantify the response facilitation or suppression at the preferred and least-preferred disparity, respectively, an FI and an SI were computed for every neuron responsive to both dichoptic and monocular gratings, at the neuron's preferred SF, defined as: 


$$
\begin{gathered}
\mathrm{FI}=\mathrm{R}_{\text {binoc_peak }} /\left(\mathrm{R}_{\text {monoc_contra }}+\mathrm{R}_{\text {monoc_ipsi }}\right) \\
\mathrm{SI}=\mathrm{R}_{\text {binoc_null }} / \max \left(\mathrm{R}_{\text {monoc_contra }}, \mathrm{R}_{\text {monoc_ipsi }}\right) .
\end{gathered}
$$

$\mathrm{R}_{\text {binoc_peak }}$ and $\mathrm{R}_{\text {binoc_null }}$ are, respectively, the largest and smallest response evoked among the eight disparities; $\mathrm{R}_{\text {monoc contra }}$ and $\mathrm{R}_{\text {monoc_ipsi }}$ are the responses to monocular gratings presented, respectively, to either the contralateral or ipsilateral eye. FI values above 1 indicate a facilitatory interaction of the eye-specific inputs, while SI values below 1 correspond to suppressive binocular interactions. Moreover, FI values above 1 and SI values below 1 in principle indicate a nonlinear integration mechanism through which responses are facilitated and suppressed, respectively. It should be borne in mind, however, that the responses measured in this study consist of the visually-evoked fluorescence signal of GCaMP6s, which provides an indirect measure of the spiking activity of neurons (Hendel et al., 2008; Grienberger and Konnerth, 2012; Lütcke et al., 2013; Rose et al., 2014). Owing to the nonlinear relationship between action potential firing and the GCaMP6 fluorescence signal, only the presence of facilitation or suppression can be reported, without inferring the precise linear/nonlinear nature of the binocular interaction shown by a cell.

\section{Disparity tuning curve fit}

To determine the width and asymmetry of disparity tuning curves obtained with $\mathrm{CRDC}$, tuning curves were fitted with an asymmetric Gaussian function using single trial responses (Hinkle and Connor, 2005), as follows:

$$
R(d)=\left\{\begin{array}{l}
R_{\text {baseline }}+R_{\text {pref }} \cdot \exp \left(-\frac{\left(d-d_{\text {pref }}\right)^{2}}{2 \sigma_{1}^{2}}\right) \text { if } d<d_{\text {pref }}, \\
R_{\text {baseline }}+R_{\text {pref }} \cdot \exp \left(-\frac{\left(d-d_{\text {pref }}\right)^{2}}{2 \sigma_{2}^{2}}\right) \text { if } d \geq d_{\text {pref }}
\end{array}\right.
$$

where $R_{\text {baseline }}$ is the baseline response, $R_{\text {pref }}$ is the response to the preferred disparity, $\sigma_{1}$ and $\sigma_{2}$ are the tuning width parameters for the left and right sides, respectively. Tuning curve width and asymmetry as plotted in Figure $7 C, D$ were quantified as $\sigma_{1}+\sigma_{2}$, and $\left|\sigma_{1}-\sigma_{2}\right|$, respectively, including only cells in which at least $50 \%$ of the tuning curve variance $\left(R^{2}\right)$ could be accounted for by the model fit.

To assess the relation between correlated and anticorrelated responses, disparity tuning curves obtained with $\mathrm{CRDC}$ or aRDC were fitted with a Gabor function using single trial responses, as follows:

$$
R(\theta)=R_{\text {baseline }}+A \cdot \exp \left(-\frac{\left(d-d_{0}\right)^{2}}{2 \sigma^{2}}\right) \cdot \cos \left(2 \pi f\left(d-d_{0}\right)+\phi\right),
$$

where $A$ is the amplitude, $d_{0}$ is the Gaussian center, $\sigma$ is the Gaussian width, $f$ is the Gabor frequency, and $\phi$ is the Gabor phase. When cells were responsive to both $\mathrm{CRDC}$ and aRDC stimuli, the tuning curves obtained with CRDC and aRDC were fitted using the same values of $R_{\text {baseline, }}, d_{0}, \sigma$, and $f$, for both curves, but different values for $A$ and $\phi\left(A_{c}, A_{a}, \phi_{c}, \phi_{a}\right.$, with the subscripts $c$ and $a$ referring to the tuning curve obtained with cRDC and aRDC, respectively; Cumming and Parker, 1997).

\section{Noise correlations}

Noise correlations were calculated between all possible pairs of disparity-tuned neurons from the same imaging plane. The single-trial $\Delta \mathrm{F} / \mathrm{F}$ responses of a given cell to dichoptic gratings were Z-scored with respect to the mean across trials. Pairwise noise correlations were then computed using the Pearson's linear correlation coefficient. While fluorescence signals were corrected for contamination by surrounding neuropil and neighboring cells, noise correlations might still be affected by potentially residual neuropil contamination. For this reason, only pairs separated by at least $20 \mu \mathrm{m}$ were considered.
Population decoding with support vector machines (SVM)

To estimate how much information about binocular disparity is carried by the joint activity of populations of neurons in each area, a population decoding approach based on SVM (Cortes and Vapnik, 1995) was employed. The decoding approach was designed to estimate which disparity, among all eight possible grating disparities, was actually presented. This discrimination among eight distinct classes was redefined as a series of binary classifications ("multiclass classification"), in which SVM were used to find the hyperplane that best separated neuronal activity data points of one class (grating disparity) from those of another class ("binary classification"). The accuracy in classifying data points of the two stimulus conditions with increasing numbers of neurons was evaluated.

The dataset consisted of populations of neurons from a given imaging plane and area, responsive to dichoptic gratings. For each neuron in the dataset, the $\Delta \mathrm{F} / \mathrm{F}_{0}$ response of each trial was split in $\mathrm{b}=6$ bins of $0.5 \mathrm{~s}$, including four bins during the stimulus period $(2 \mathrm{~s})$ and two bins immediately following it, and the mean $\Delta \mathrm{F} / \mathrm{F}_{0}$ of each bin was taken as one activity data point. As such, for each neuron and disparity, there were ap activity points, with ap $=\mathrm{t} \times \mathrm{b}$, where $t=6$ trials for each disparity and $\mathrm{b}=6$ bins.

For each decoding session, a subpopulation of $\mathrm{N}$ neurons was randomly sampled from a given population of neurons recorded in the same imaging plane and area. A matrix of data points was constructed, with $\mathrm{N}$ columns (neurons, corresponding to the "features") and ap $\times \mathrm{d}$ rows (activity points $\times$ disparities, corresponding to the "observations"). The data matrix was divided into two separate sets, a training set and a test set. The training set included $0.9 \times$ ap randomly chosen activity points for each disparity; the test set included the remaining $0.1 \times$ ap activity points ("10-fold cross-validation"). A multiclass decoder was constructed by training 28 distinct binary classifiers, each considering only two different disparities as the two classes, and exhausting all combinations of disparity pairs ("one-vs-one"). The identity of each observation of the training set was also provided to every classifier ("supervised classification"). Then, the multiclass decoder was probed on the test set. Each observation of the test set was evaluated by each of the 28 binary classifiers to predict its class (disparity). The class identity that was more frequently predicted across the 28 classifications was taken as the predicted class identity of that observation. This evaluation was performed for every observation of the test set. The procedure was then repeated on a different training set and test set, across all 10 -folds, to produce an average accuracy estimate of the decoder for a given subpopulation of $\mathrm{N}$ neurons. Twenty different random resamplings of $\mathrm{N}$ neurons from the population were performed and the outcomes were averaged to generate a measure of decoding performance of a given $\mathrm{N}$ for each imaging plane, as reported in Figure 6. An alternative multiclass decoding procedure was additionally tested, in which the binary classification was performed between one disparity and all other disparities as a single class ("one-vsall"). Likely owing to the imbalanced number of data points between one-vs-all classes, the decoding performance of this alternative procedure was slightly worse, although qualitatively similar, than the one-vsone classifier (data not shown). We hence chose to perform population decoding based on one-vs-one classifiers.

Significance levels for classification accuracy were determined by using a similar decoding procedure but training decoders on training sets in which the identities of the observations were randomly shuffled, repeating the shuffling 100 times for each imaging plane separately and for each of the 20 resamplings of $\mathrm{N}$ neurons. The significance level was finally determined for each $\mathrm{N}$ of neurons as the 99.95th percentile across all shuffles $(p=0.001)$. The binary classifiers consisted of SVM with a linear kernel. The decoding procedures were performed using custom-written routines based on the function fitecoc (kernel scale, 1; box constraint, 1) as part of the Statistics and Machine Learning Toolbox in MATLAB (MathWorks).

\section{Statistical analyses}

All data and statistical analyses were performed using custom-written MATLAB code (MathWorks). Sample sizes were not estimated in advance. No randomization or blinding was performed during 
experiments or data analysis. Data are reported as mean with standard error of the mean (mean \pm SEM). Data groups were tested for normality using the Shapiro-Wilk test in combination with a skewness test and visual assessment (Ghasemi and Zahediasl, 2012). Comparisons between data groups where made using the appropriate tests: one-way ANOVA, Kruskal-Wallis test, circular non parametric multisample test for equal medians (Fig. 8F; Berens, 2009). For multiple comparisons, Bonferroni correction was used. All tests were two-sided. Significance levels for spatial clustering in Figure $4 B$ were determined by permutation tests. In each permutation ( $n=1000$ permutations), the $x y$ positions of all neurons from a given area were randomly shuffled and the mean (across all disparity-tuned neurons of a given area) difference in disparity preference for each distance bin was calculated; $95 \%$ confidence intervals were computed as $2.5-97.5$ th percentiles of all permutations. Significance levels for noise correlations in Figure 5C, $D$ were determined by permutation tests. In each permutation ( $n=1000$ permutations), noise correlations of all pairs of disparity-tuned neurons from each imaging plane of a given area were randomly shuffled and the mean (across planes) noise correlations for each bin of distance (Fig. $5 C$ ) or bin of difference in disparity preference (Fig. 5D) were calculated; 95\% confidence intervals were computed as $2.5-97.5$ th percentiles of all permutations. The statistical significances are reported in the figures, with asterisks denoting significance values as follows: ${ }^{\star} p<0.05,{ }^{* *} p<0.01,{ }^{* *} p<0.001$.

\section{Data and code availability}

Data generated during this study are available online at https://edmond. mpdl.mpg.de/imeji/collection/_chMNO_mc1EQ5Gu5 in the form of processed data (extracted fluorescence time courses). The program code used to perform data analyses and to reproduce figures is available at https://github.com/lachioma/LaChioma_et_al_2020. Raw two-photon imaging data have not been deposited because of file size but are available on reasonable request. Additional requests for data and code should be directed to the corresponding author.

\section{Results}

To investigate binocular integration and disparity selectivity across mouse visual cortex, we performed in vivo two-photon calcium imaging in V1, and areas LM and RL. We focused on these three areas since they contain the largest, continuous cortical representation of the central, binocular region of the visual field.

\section{Identification and targeting of areas V1, LM, and RL for two-photon imaging}

To localize areas V1, LM, and RL for subsequent two-photon imaging, we used intrinsic signal imaging to map the overall retinotopic organization of the visual cortex (Fig. 1A; Kalatsky and Stryker, 2003; Marshel et al., 2011). Continuously drifting, horizontally and vertically moving bar stimuli were employed to generate two orthogonal retinotopic maps with precise vertical and horizontal meridians. By using the established visual field representations in mouse visual cortex (Marshel et al., 2011; Garrett et al., 2014), the boundaries among areas V1, LM, and RL could be readily identified (Fig. $1 B, C$ ).

After having identified V1, LM, and RL, we targeted these areas for two-photon imaging (Fig. 1E,F). Visually-evoked activity of individual neurons was measured using the genetically encoded calcium indicator GCaMP6s (Chen et al., 2013), coexpressed with the structural marker mRuby2 (Rose et al., 2016). The latter aided image registration for correcting motion artifacts and improved the identification of neurons for marking ROIs.

\section{Binocular disparity is encoded by large fractions of neurons in areas $\mathrm{V1}, \mathrm{LM}$, and $\mathrm{RL}$}

Disparity tuning was characterized using drifting vertical gratings displayed in a dichoptic fashion at varying interocular disparities (Fig. 1D,F). Eight different grating disparities were generated by systematically varying the relative phase between the gratings presented to either eye, while drift direction, speed, and spatial frequency were kept constant across eyes. In addition to such "dichoptic gratings," gratings were also displayed to each eye separately ("monocular gratings") to determine OD and compare monocular with binocular responses.

To mitigate the "correspondence problem" that arises when using circularly repetitive stimuli, dichoptic grating stimuli were presented at a low spatial frequency $\left(0.01\right.$ cycles $/{ }^{\circ}$, i.e., $100 \%$ cycle), such that for most cells no more than a single grating cycle is covered by individual RFs, given the typical RF size in mouse visual areas (Van den Bergh et al., 2010; Smith et al., 2017) and a binocular overlap of $\sim 40^{\circ}$ (Scholl et al., 2013; Sterratt et al., 2013). While gratings matched to the scale of the RF do not fully eliminate the correspondence problem, they allow a more reliable measurement of disparity tuning curves than would be possible at high spatial frequencies.

Across areas, $\sim 15 \%$ of neurons were responsive (see Materials and Methods) to vertical, dichoptic gratings (percentage of responsive cells, mean \pm SEM across planes, V1, $14.1 \pm 2.0 \%$; LM, $15.8 \pm 1.4 \%$; RL, $19.0 \pm 0.9 \%)$. Altogether, the mean response magnitude was similar across areas $(\Delta \mathrm{F} / \mathrm{F}$, mean \pm SEM across planes, V1, $57 \pm 3 \%$; LM, $65 \pm 6 \%$; RL, $55 \pm 3 \%$ ). For each responsive cell, a disparity tuning curve was computed by plotting its average calcium response in function of the interocular disparity of the dichoptic gratings (Fig. 2A). Typically, across areas, disparity tuning curves of neurons showed a strong modulation. To quantify the magnitude of modulation caused by binocular disparity, a DI, based on the vectorial sum of responses across disparities (Scholl et al., 2013; La Chioma et al., 2019), was calculated for each cell, with values closer to one for highly selective cells and values closer to zero for less selective cells. Cells with DI $>0.3$ were defined as disparity-tuned.

To determine the overall sensitivity for binocular disparity across the three areas, the distribution of DI values of all cells was plotted (Fig. 2B). In all areas, the majority of neurons showed at least some degree of modulation to binocular disparity (percentage of responsive cells defined disparity-tuned, mean \pm SEM across planes, V1, $89.0 \pm 1.3 \%$ : LM, $88.4 \pm 2.7 \%$; RL, $88.3 \pm 2.1 \%)$. Using more stringent criteria for defining responsive cells did not qualitatively change the DI distribution for each area (data not shown). Across all three areas, we observed overall similar degrees of disparity selectivity (Kruskal-Wallis test across planes, $\chi^{2}(2)=1.9773, p=0.3721 ; \mathrm{V} 1, n=8$ imaging planes, 600 responsive cells total, 7 mice; LM, $n=7$ imaging planes, 550 responsive cells total, 7 mice; RL, $n=6$ imaging planes, 547 responsive cells total, 5 mice; Fig. $2 B$ ). In addition, all three areas showed continuous DI distributions, indicating a continuum of disparity tuning, without pointing to the presence of a distinct subset of highly tuned cells. Thus, disparity sensitivity to gratings is widespread in V1 and higher areas LM and RL.

\section{OD is similar across visual areas and is not correlated with disparity selectivity}

By definition, disparity-tuned neurons are binocular. However, conventionally, a neuron's binocularity is assessed by measuring its OD based on responses to monocular stimuli only. How are disparity selectivity and OD, which represent two different ways of describing a neuron's binocularity, related to each other? To address this question, we first computed the ODI (ranging from +1 or -1 ) using the neuronal responses to monocular gratings presented to each eye separately. As reported (Dräger, 1975; 
Gordon and Stryker, 1996; Mrsic-Flogel et al., 2007; Rose et al., 2016), the distribution of ODI values for mouse $\mathrm{V} 1$ is considerably biased toward the contralateral eye (Fig. 3A). Neurons in areas LM and RL, for which OD measurements have not been reported yet, were also more strongly driven by the contralateral eye and showed ODI distributions comparable to V1 (ODI median, V1: 0.40, LM: 0.40, RL: 0.44; ODI mean \pm SEM across experiments, V1: $0.17 \pm 0.07, \mathrm{LM}: 0.25 \pm 0.06$, RL: $0.21 \pm 0.09$; Kruskal-Wallis test for medians across planes $\chi^{2}(2)=0.84, p=0.658$ ).

We then analyzed the relationship between OD and disparity selectivity by plotting DI values against ODI values for individual cells in each area. Disparity-tuned neurons homogeneously covered the entire range of ODI values (Pearson's correlation, $\mathrm{V} 1: r=-0.03, p=0.706 ; \mathrm{LM}: r=0.07$, $p=0.390$; RL: $r=-0.10, p=0.095$; Kruskal-Wallis test across ODI bins: V1, $\chi^{2}(6)=4.5563, p=0.6018$; LM, $\chi^{2}(6)=8.1326, p=0.2286$; RL, $\chi^{2}(6)=3.0209$, $p=0.8062$; Fig. $3 B$ ). Notably, neurons classified as monocular by $\mathrm{OD}$ measurements $(\mathrm{ODI} \approx 1$ or ODI $\approx-1$ ) could be disparity-tuned, hence clearly reflecting integration of inputs from both eyes. Thus, there is no clear relationship between OD and disparity selectivity, in line with other studies in mice (Scholl et al., 2013), cats (Ohzawa and Freeman, 1986; LeVay and Voigt, 1988), and monkeys (Prince et al., 2002a; Read and Cumming, 2004).

\section{Most neurons exhibit strong disparity- dependent facilitation and suppression} To examine the integration of visual inputs from both eyes, we next compared the responses evoked by dichoptic and monocular gratings. The response at the optimal binocular disparity was generally much larger than the sum of the two monocular responses for most neurons, indicating a robust facilitatory effect of binocular integration (Fig. $3 C$ ). At the same time, there were also strong suppressive binocular interactions: at the least effective disparity, the response was generally absent or smaller than the larger of the two monocular responses (Fig. 3C).

To quantify the response facilitation or suppression at the preferred and least-preferred disparity, respectively, a FI and a SI was computed for every neuron responsive to both dichoptic and monocular gratings (see Materials and Methods). FI values above 1 indicate a facilitatory interaction of the eye-specific inputs, while SI values below 1 correspond to suppressive binocular interactions. Most neurons across the three areas were overall highly and similarly susceptible to binocular interactions, as inputs from both eyes were integrated with strong response facilitation as well as suppression as a function of binocular disparity (one-way ANOVA for FI across areas: $F_{(2,18)}=0.4353, p=0.6537$; SI, $F_{(2,18)}=1.3776, p=0.2775$; Fig. $\left.3 C\right)$.

\section{Spatial clustering of neurons with similar disparity preference}

While no large-scale spatial arrangement for response properties has been observed in mouse visual cortex (Mrsic-Flogel et al., 2007; Zariwala et al., 2011; Montijn et al., 2014), several studies
B
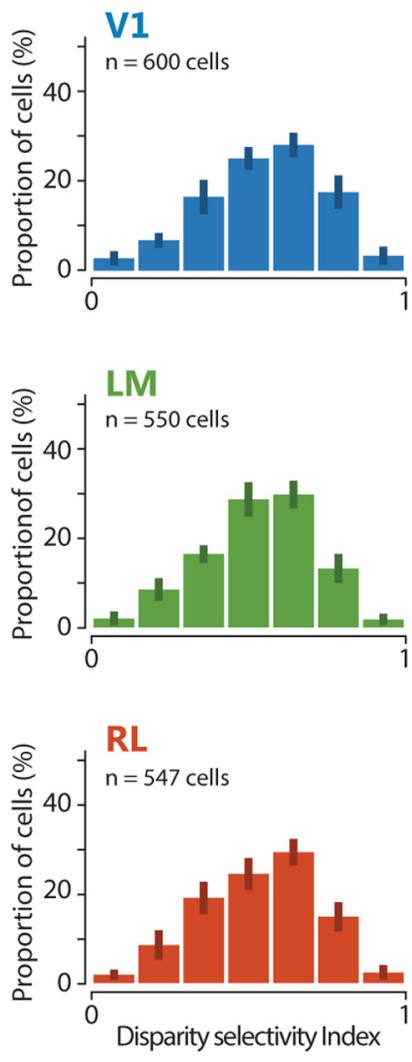

Figure 2. Functional characterization of disparity-tuned neurons in areas V1, LM, and RL using dichoptic gra作 Grating disparities
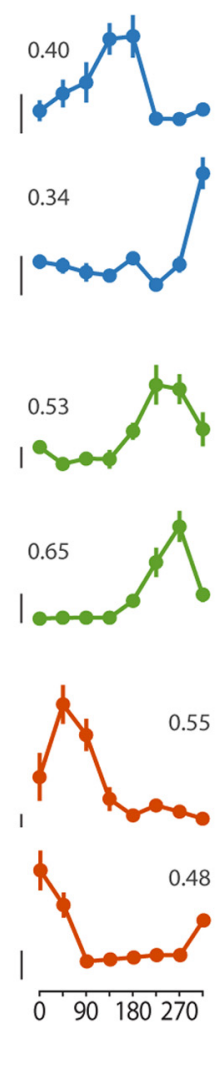

\section{gra-
LM,
ating
onse
B,
B.}

have reported a fine-scale organization for orientation preference and OD (Ringach et al., 2016; Kondo et al., 2016; Maruoka et al., 2017; Scholl et al., 2017). To test whether binocular disparity preference is organized in a similar fashion, we generated colorcoded, disparity maps for each imaging plane, with a cell body's hue coding for its disparity preference (Fig. 4A). As expected, inspection of these maps did not reveal a large-scale arrangement of disparity-tuned neurons.

To investigate whether a spatial organization exists on a finer scale, we plotted the difference in disparity preference between every pair of cells as a function of their cortical distance (Fig. $4 B$ ). None of the three areas showed a clear dependence of tuning similarity on cortical distance, indicating a lack of a largescale spatial arrangement of disparity-tuned cells. Nonetheless, adjacent neurons, located within $10 \mu \mathrm{m}$ from each other, showed a preference for similar disparities, indicating some degree of spatial clustering on the scale of $10 \mu \mathrm{m}$. This spatial scale is consistent with values reported for orientation and spatial frequency tuning in mouse visual cortex (on the scale of $\sim 35 \mu \mathrm{m}$; Ringach et al., 2016; Scholl et al., 2017), and also consistent with reports demonstrating a fine-scale organization for orientation tuning and $\mathrm{OD}$ (in the range of 5-20 $\mu \mathrm{m}$ ), with neurons sharing functional properties arranged into microcolumns (Kondo et al., 2016; Maruoka et al., 2017). 
A

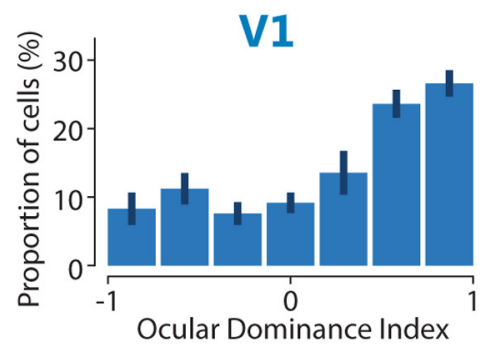

B

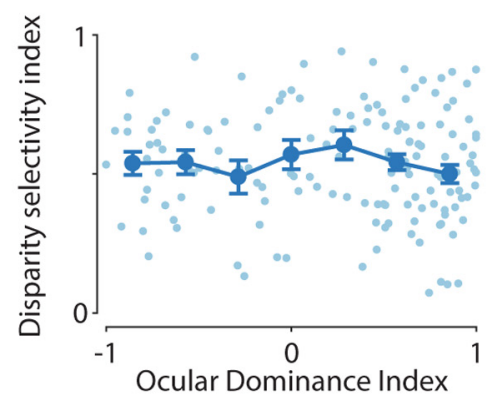

C
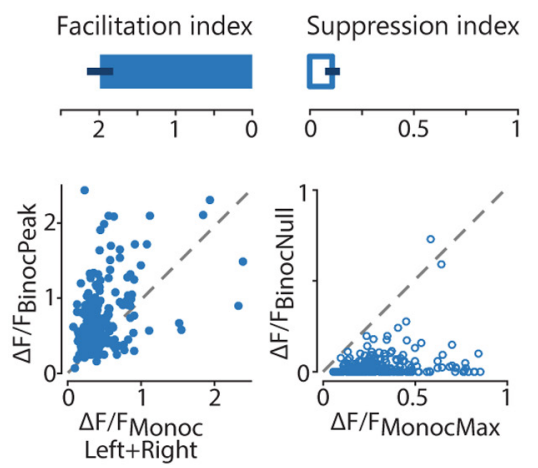
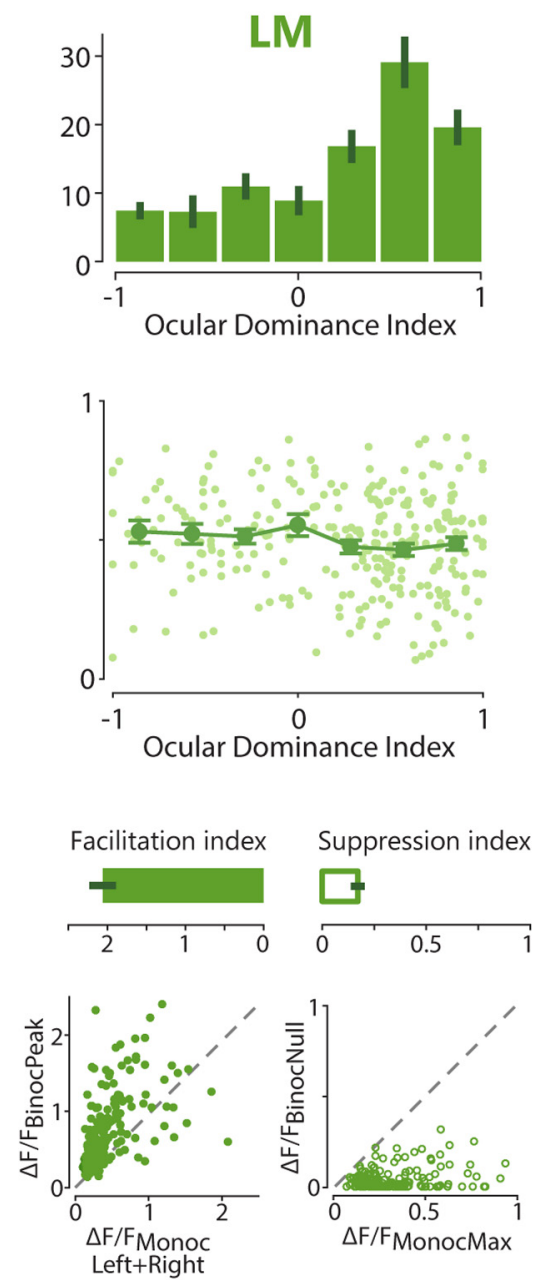
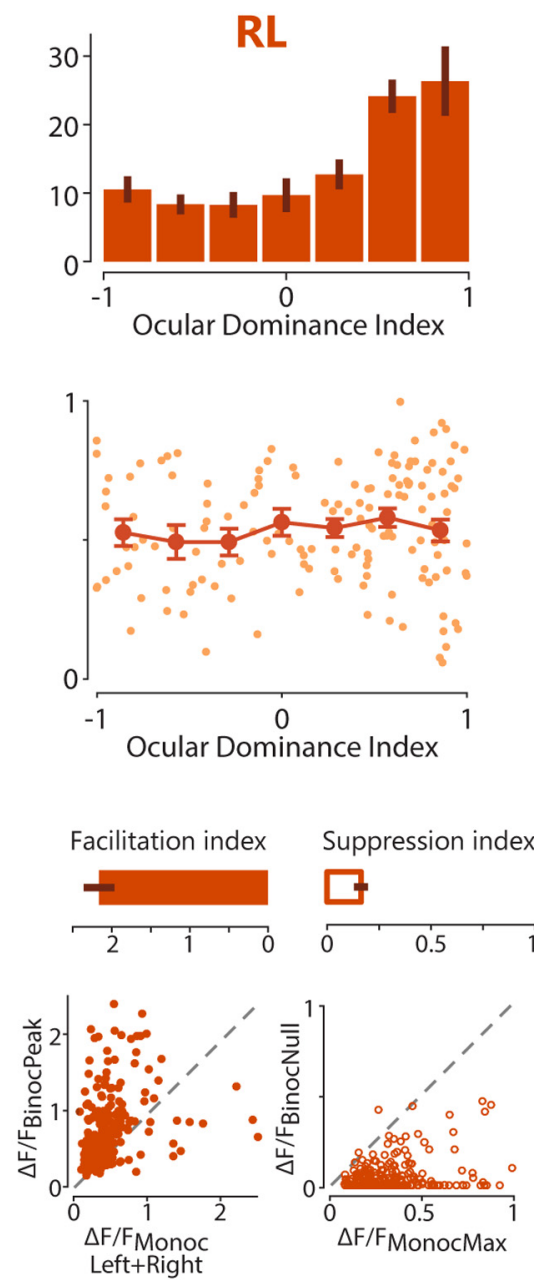

Figure 3. $\mathrm{OD}$ and binocular interaction of individual neurons across visual areas. $\boldsymbol{A}, \mathrm{ODI}$ distributions in areas $\mathrm{V} 1, \mathrm{LM}$, and RL, with bar plots indicating mean \pm SEM across imaging planes. $\boldsymbol{B}$, Scatter graphs plotting the relationship between ODI and DI. Lines with error bars plot the mean \pm SEM across neurons, with individual cells represented by dots in lighter shading. $\boldsymbol{C}$, Facilitatory and suppressive interactions upon binocular stimulation. Top panels, Bar plots of facilitation and suppression indices for each area, with mean \pm SEM across imaging planes. Bottom panels, For each area, the graph on the left plots the strongest response to dichoptic (binocular) gratings $\left(\Delta \mathrm{F} / \mathrm{F}_{\text {BinocPeak }}\right)$ against the sum of the strongest contralateral and ipsilateral responses $\left(\Delta F / F_{\text {Monocleft + Right }}\right)$ for individual neurons, showing a strong overall response facilitation with binocular stimulation at the preferred disparity. For each area, the graph on the right plots the weakest response to dichoptic (binocular) gratings $\left(\Delta \mathrm{F} / \mathrm{F}_{\text {BinocNull }}\right)$ against the strongest monocular response $\left(\Delta \mathrm{F} / \mathrm{F}_{\text {MonocMax }}\right)$ for individual neurons, showing a strong overall response suppression with binocular stimulation at the least-preferred (null) disparity.

\section{Non-uniform distribution of disparity preference in individual experiments}

While there was no obvious, large-scale spatial organization for disparity preference, in each individual experiment we found that disparity preferences showed a non-uniform distribution, with a peak characteristic for each experiment (Fig. 4A). This peak disparity varied over the whole range from experiment to experiment, showing no systematic relationship across experiments or animals. We consider it unlikely that the variation of apparent population peak disparity across mice reflects a true biological phenomenon. Most likely, these variations across experiments reflect differences in the alignment of the optical axes of the animal's eyes, created mostly by a technical factor: namely the precise positioning of the haploscope apparatus, which could not be controlled with the necessary precision from experiment to experiment. It follows that the disparity preference distribution in each experiment is likely related to the actual optical axes of the mouse eyes in that imaging session, with the population peak disparity being approximately aligned with the visual field location of retinal correspondence between eyes (La Chioma et al., 2019). To determine the overall range of binocular disparity preferences in each area, the disparity preference distributions of individual experiments were aligned by setting the population peak arbitrarily to $180^{\circ}$ phase and averaging the distributions across experiments (Fig. 4C). We observed that the disparity preference distributions were overall comparable across areas. The relatively small variability across individual experiments indicates similarly peaked distributions across experiments and mice, likely reflecting the overrepresentation of neurons approximately tuned to retinal correspondence, and supporting our interpretation that the shift in the peak from experiment to experiment is caused by imperfect alignment of the haploscope apparatus.

\section{Noise correlations are higher between neurons with similar disparity preference}

We next analyzed the trial-to-trial fluctuations in response strength between pairs of neurons, the so-called noise correlations. The amount of variability shared between neurons has implications for neural coding, and is assumed to reflect 
connectivity among cells (Averbeck et al., 2006; Cohen and Kohn, 2011; Ko et al., 2011; Schulz et al., 2015; Kohn et al., 2016), with highly correlated neurons being more strongly interconnected or sharing more common inputs compared with neurons with lower noise correlations (Ko et al., 2011). Over the entire populations, in each area, the pairwise noise correlations were on average weak but significantly larger than zero (one sample $t$ test against mean $=0, \mathrm{~V} 1, p=0.0048, \mathrm{LM}$, $p=0.0018$, RL, $p=0.0028$; Fig. $5 A$, insets), in line with previous reports in mouse visual cortex (Ko et al., 2011; Montijn et al., 2014; Rose et al., 2016; Khan et al., 2018). Moreover, the distributions of noise correlations were comparable across areas (oneway $\left.\operatorname{ANOVA,} F_{(2,18)}=1.0054, p=0.3855\right)$, and all showed a positive tail consisting of small numbers of highly correlated pairs (Fig. 5A).

It has been previously shown that the connection strengths between neurons in mouse visual cortex is stronger for cells with similar RFs and orientation tuning (Cossell et al., 2015), or for cells with high noise correlations (Ko et al., 2011), but it is not influenced by cortical distance (Cossell et al., 2015). Do nearby neurons or similarly disparity-tuned neurons have higher noise correlations? To answer this question, for each pair of disparity-tuned neurons, the value of their noise correlation was related to both their cortical distance and their disparity preference (Fig. 5B-D). Nearby and distant neurons showed comparable noise correlations, indicating no dependence of noise correlations on cortical distance (Fig. 5C). In contrast, pairs with a similar disparity preference $\left(<45^{\circ}\right.$ phase) showed substantially higher noise correlations compared with pairs with dissimilar preference (Fig. 5D). Thus, neurons with similar disparity preference are more strongly interconnected or share common inputs.
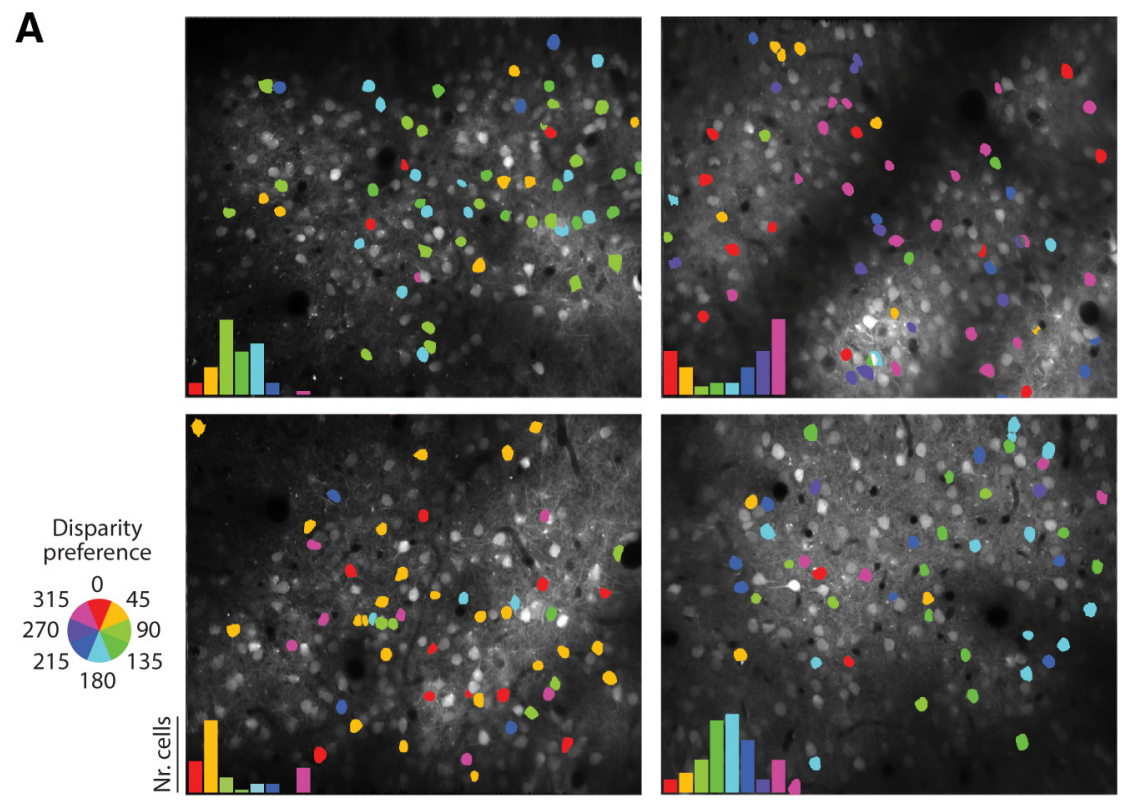

B
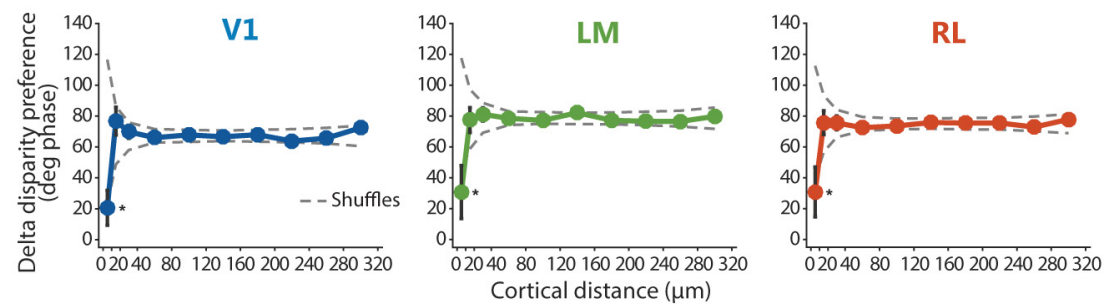

C
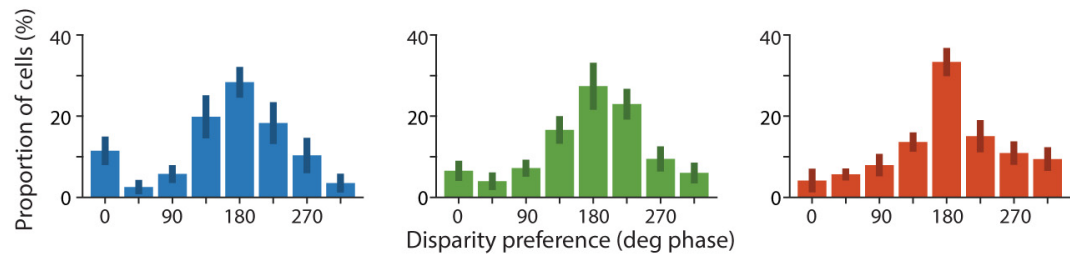

Figure 4. Spatial and functional organization of disparity-tuned neurons. $\boldsymbol{A}$, Example disparity maps from four different imaging planes, with neurons color-coded for disparity preference. Insets, disparity preferences show non-uniform distributions, with a population peak disparity characteristic for each individual experiment. $\boldsymbol{B}$, Spatial organization for disparity tuning. Difference in disparity preference between every pair of disparity-tuned cells in each imaging plane plotted as a function of the cortical distance between cells. Plot lines and error bars indicate mean \pm SEM across neurons. The dashed lines indicate the $95 \%$ confidence interval, as determined by random shuffles of disparity preferences and cell $x, y$ positions in each imaging plane ( ${ }^{*} p<0.05$ at the $10-\mu \mathrm{m}$ cortical distance bin). C, Peak aligned distributions of disparity preferences, averaged across experiments (V1, $n=8$ imaging planes, 536 disparity-tuned cells total, 7 mice; LM, $n=7$ imaging planes, 484 disparity-tuned cells total, 7 mice; RL, $n=6$ imaging planes, 480 disparity-tuned cells total, 5 mice). The population peak was arbitrarily set to $180^{\circ}$ phase.

\section{Neuronal populations across visual} areas effectively discriminate between grating disparities Accurate representations of binocular disparity are likely encoded at the population level, since individual neurons are insufficient for this task, considering the narrow range of their response properties (Scholl et al., 2013; Burge and Geisler, 2014; Kato et al., 2016). Having shown that large numbers of individual neurons in all three areas encode binocular disparity, we next investigated how much information is carried, in each area, by the joint activity of multiple neurons.

We therefore employed a population decoding approach based on SVM (Cortes and Vapnik, 1995), trained using the calcium transients of populations of neurons. For each area, the SVM decoders were used to estimate, on a trial-to-trial basis, which of the eight grating disparities was actually presented (see
Materials and Methods). Across areas, decoders were able to effectively estimate binocular disparity, since populations with as few as two neurons allowed significantly correct prediction of stimulus disparity, with initially steep improvement with increasing population sizes (Fig. 6). The three areas showed a similar capacity of discriminating disparity over the entire range of population sizes tested (Fig. 6). Moreover, a decoder built on a pseudo-population consisting of neurons pooled together from all three areas indistinctly, showed a curve of discrimination accuracy comparable to decoders trained on populations from each area separately (data not shown), thereby indicating that neurons from the different areas were interchangeable from the perspective of the decoder and hence contributed similarly to decoding. Together, these data indicate that populations of neurons in areas V1, LM, and RL efficiently encode binocular 

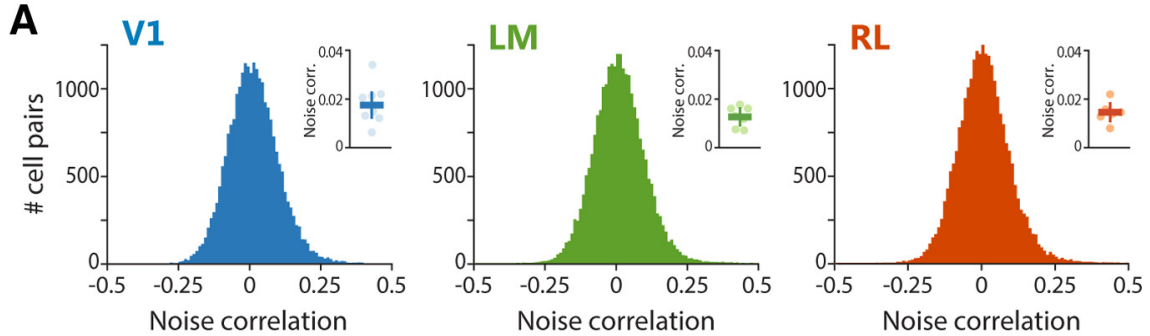

B
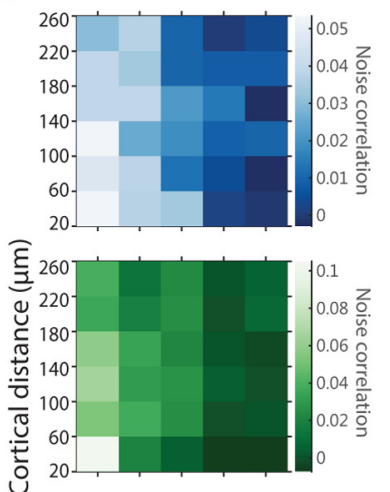

c

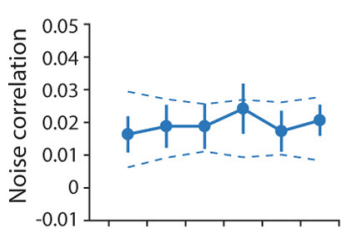

D
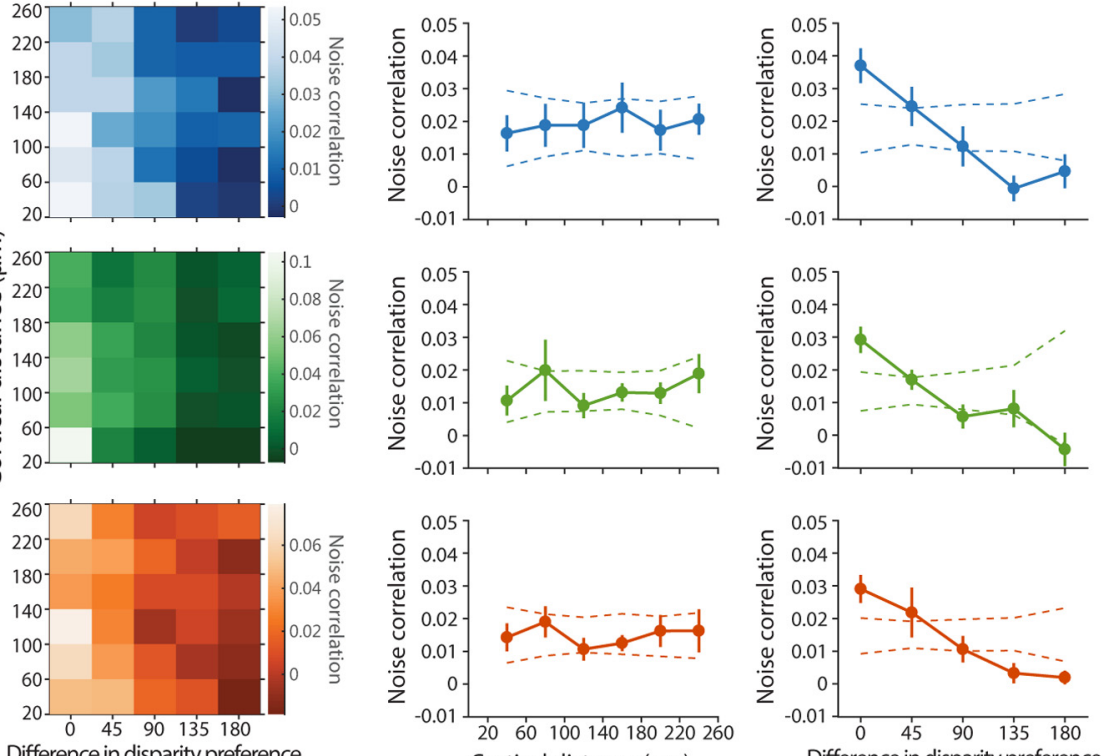

(deg phase)
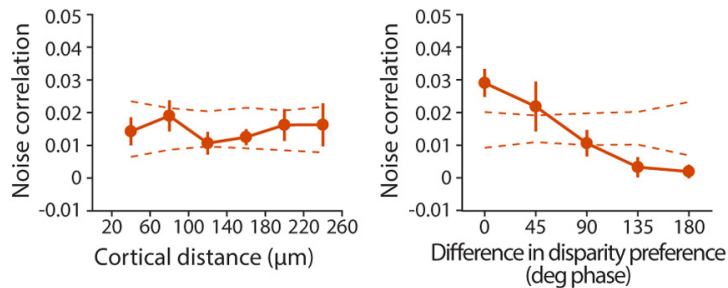

Figure 5. Noise correlations are higher between neurons with similar disparity preference. $\boldsymbol{A}$, Distributions of pairwise noise correlations. Only one distribution for each area is shown as an example. Note the small positive tail in the distributions. Insets, Pairwise noise correlations averaged across planes \pm SEM, with individual planes indicated with circles in lighter shading. $\boldsymbol{B}$, Dependence of noise correlations on both cortical distance and difference in disparity preference between each cell pair. $\boldsymbol{C}$, Pairwise noise correlation as a function of cortical distance between each cell pair. $\boldsymbol{D}$, Pairwise noise correlation as a function of difference in disparity preference between each cell pair. In $\boldsymbol{C}, \boldsymbol{D}$, plot lot lines and error bars indicate mean \pm SEM across imaging planes, and dashed lines indicate the $95 \%$ confidence interval, as determined by random shuffles of cell x,y positions in each imaging plane. For computing pairwise noise correlations, only cell pairs separated by at least $20 \mu \mathrm{m}$ were considered.

disparity and can effectively discriminate between grating disparities, with a comparable accuracy across areas.

\section{Disparity tuning curves differ among visual areas}

In primates, neurons in extrastriate areas show broader disparity tuning, and tuning curves are more often asymmetric compared with those in V1 (Cumming and DeAngelis, 2001; Prince et al., 2002b; DeAngelis and Uka, 2003). Tuning curve symmetry has been taken as an indicator for distinguishing between two different mechanisms underlying disparity sensitivity, the positionshift and the phase-shift mechanism (see Discussion; Qian, 1997; Cumming and DeAngelis, 2001; Tsao et al., 2003). We thus characterized the shape of disparity tuning curves in mouse visual cortex, using RDC (Julesz et al., 1980; see Fig. 8A, top), in awake animals. RDC allowed measuring absolute disparities and characterizing tuning as a function of visual angle, without the ambiguity deriving from the inherent circularity of gratings. Stimulus presentation in awake mice resulted in substantially stronger neuronal responses to RDC compared with the anesthetized state, activating a higher fraction of neurons and with larger calcium transients on average (data not shown), while preserving the overall disparity tuning of individual neurons across states (La Chioma et al., 2019). Many neurons across areas exhibited clear responses to RDC, with reliable activation by a limited range of disparities (Fig. 7A). Compared with dichoptic gratings as measured in anesthetized mice, RDC presented to awake mice activated a much higher proportion of cells (percentage of cells responsive to RDC, mean \pm SEM across planes, V1, $47.2 \pm$ 3.6\%; LM, $44.4 \pm 6.6 \%$; RL, $32.9 \pm 2.5 \%$ ). When comparing the fraction of responsive cells between experiments with grating and RDC stimuli, it should be borne in mind that ROI segmentation was performed with two different methods, namely manual segmentation for gratings and automated segmentation for RDC (see Materials and Methods).

The disparity tuning curves of individual neurons as measured with RDC showed a variety of shapes (Fig. 7A). To analyze tuning curve shapes in more detail, we fitted them with an asymmetric Gaussian function, with separate width parameters for the left $(\sigma 1)$ and right side $(\sigma 2$; Fig. $7 B$; Hinkle and Connor, 2005; for details, see Materials and Methods), allowing to assess potential tuning curve asymmetries. Among all disparity-tuned cells, tuning width $(\sigma 1+\sigma 2)$ and asymmetry $(|\sigma 1-\sigma 2|)$ varied over a large range (Fig. $7 C, D)$. On average, neurons in LM and RL had significantly wider tuning curves compared with V1 (mean of medians across planes \pm SEM, V1, $7.18 \pm 0.66^{\circ}$; LM, $11.13 \pm 0.69^{\circ}$; RL, $11.20 \pm 0.76^{\circ}$; KruskalWallis test across planes, $\chi^{2}(2)=12.201$, $p=2.242 \mathrm{e}-03$; post hoc Bonferroni-corrected Mann-Whitney tests, V1 vs LM, $p=0.02369 ; \mathrm{V} 1$ vs RL, $p=8.638 \mathrm{e}-04 ; \mathrm{LM}$ vs $\mathrm{RL}$, unadjusted $p=0.888 ; \mathrm{V} 1, n=9$ imaging planes; LM, $n=8$ planes; RL, $n=9$ planes; Fig. $7 C$ ). Likewise, tuning curves in RL were more asymmetric than in $\mathrm{V} 1$, a difference that was also visible in $\mathrm{LM}$ as a trend (mean of medians across planes $\pm \mathrm{SEM}, \mathrm{V} 1$, $2.78 \pm 0.44^{\circ} ; \mathrm{LM}, 4.24 \pm 0.37^{\circ} ; \mathrm{RL}, 5.40 \pm 0.46^{\circ}$; Kruskal-Wallis test across planes, $\chi^{2}(2)=12.286, \quad p=2.148 \mathrm{e}-03$; post hoc Bonferroni-corrected Mann-Whitney tests, V1 vs LM, $p=0.06195$; V1 vs RL, $p=8.638 \mathrm{e}-04$; LM vs RL, $p=0.708$; Fig. $7 D$ ).

A potential confounder of these findings is that areas V1, LM, and RL contain partially different visual field representations. The three areas have similar azimuthal representations of the binocular visual field, but different ones in elevation (Garrett et al., 2014; Zhuang et al., 2017): V1 covers both the lower and the upper visual field, while LM and RL represent mainly the upper and lower visual field, respectively. It is hence possible that the shape of disparity tuning curves is related to retinotopic elevation, similarly to other visual response properties (Aihara et al., 2017; La Chioma et al., 2019; Sit and Goard, 2020), rather than arising from area-specific mechanisms. Thus, we tested whether there is any relationship between tuning curve shape and RF elevation of individual cells. In none of the three areas did we find a 
relationship of tuning width or asymmetry with visual field elevation (data not shown).

Altogether, disparity-tuned neurons have broader and more asymmetric tuning curves in mouse higher visual areas compared with V1, similar to what has been observed in primate visual cortex. These areal differences in disparity tuning shape result from area-specific processing, rather than being related to differences in visual field representation.

\section{Area-specific responses to aRDC}

To extract depth information using binocular disparity, the visual system must determine which points in the left-eye and right-eye images correspond to the same visual feature among a number of potential false matches, i.e., the correspondence problem must be solved. Neurons sensitive to true stereo-correspondence should respond only to correct, but not false matches. Any sensitivity to false matches would indicate that further processing is needed for achieving true stereo-correspondence, e.g., in downstream areas. In primates, comparing the sensitivity to true and false matches across visual areas helped delineating their hierarchy and role for stereo-based depth processing (Parker, 2007). To test this in mouse visual cortex, we compared disparity tuning in response to binocularly correlated and anticorrelated RDC, in which corresponding dots between the two eyes have opposite contrast that generate false matches (Fig. 8A; Cumming and Parker, 1997). On average, $48.1 \%, 78.7 \%$, and $55.7 \%$ of responsive neurons in areas V1, LM, and RL, respectively, were disparity-tuned to cRDC (see Materials and Methods). In contrast, aRDC stimuli activated far fewer neurons (V1: 15.7\%, LM: 7.6\%, RL: 13.6\%). Across areas, $3-4 \%$ of all responsive cells were disparity-tuned to both cRDC and aRDC (Fig. 8C). Notably, LM showed a significantly higher proportion of cells tuned only to cRDC compared with V1 and $\mathrm{RL}$, while having a smaller proportion of cells tuned only to aRDC (Fig. 8C). Using more stringent criteria for defining responsive cells did not qualitatively change the proportions of disparity-tuned cells (data not shown). As for disparity tuning curve shape, we found no relationship between sensitivity to aRDC and visual field elevation (data not shown).

Neurons tuned to both cRDC and aRDC often exhibited a tuning inversion: disparities evoking strong responses with correlated stimuli caused weak activations with anticorrelated stimuli and vice versa (Fig. $8 B$ ). The tuning inversion indicates that these neurons operate only as local disparity detectors, disregarding contrast sign and the global consistency of image features between the two eyes, thereby resulting in responses to false matches (Cumming and Parker, 1997). To quantitatively describe the relation between correlated and anticorrelated responses, we fitted the disparity tuning curves of each neuron with Gabor functions (Fig. 8D; Cumming and Parker, 1997; for details, see Materials and Methods). The offset in Gabor phase between $\mathrm{CRDC}$ and $\mathrm{aRDC}$ tuning curves quantifies the degree of tuning inversion, with values of $180^{\circ}$ phase indicating full inversion and values around $0^{\circ}$ phase indicating no change. Likewise, the ratio of the Gabor amplitude between aRDC and cRDC tuning curves quantifies the change in disparity sensitivity, with values below 1 signifying a reduced response modulation to aRDC relative to $\mathrm{CRDC}$. Plotting these two measures against each other reveals that most cells across areas underwent tuning inversion (phase offset of around $180^{\circ}$ phase) and a decrease in modulation to aRDC (amplitude ratio $<1$; Fig. $8 E$ ). While the incidence of tuning inversion is similar across all three areas (circular non parametric multisample test for equal medians, across cells, $p=0.1889$; Fig. $8 F$ ), area LM shows a significantly lower amplitude ratio (Kruskal-Wallis test across cells, $\chi^{2}=26.987$, $p=1.3800-06$; post hoc Bonferroni-corrected Mann-Whitney tests, V1 vs $\mathrm{LM}, p=1.493 \mathrm{e}-05$; V1 vs RL, unadjusted $p=0.5372$; LM vs RL, $p=3.981 \mathrm{e}-05$; sign test against median $=1$, across cells: V1, $p=1.308-04$; LM, $p=1.514 \mathrm{e}-09$; RL, $p=0.1102$; Fig. $8 G)$. Thus, area LM features a smaller fraction of neurons tuned to aRDC, and these neurons display weaker disparity modulation to aRDC, compared with V1 and RL. We conclude that mouse area LM performs a higher-level analysis of disparity signals compared with V1 and RL.

\section{Discussion}

Our study shows that the integration of signals from both eyes is a prominent feature of mouse visual cortex. Large fractions of neurons in areas V1, LM, and RL, even when classified as monocular by conventional OD measurements, are in fact binocular, in the sense that their activity can be strongly facilitated or suppressed by simultaneous input from both eyes and over a specific range of interocular disparities. We observed some degree of fine-scale spatial organization for disparity tuning, as neurons with similar disparity preference are clustered within a horizontal range of $\sim 10 \mu \mathrm{m}$. Moreover, similarly tuned neurons have higher noise correlations, suggesting that they are more strongly interconnected or share common input. Neurons in higher areas LM and RL showed broader and more asymmetric disparity tuning curves compared with V1. Comparing responses to binocularly correlated and anticorrelated RDC, we found that area LM shows a higher selectivity for correlated stimuli, while being less sensitive to aRDC.

\section{Disparity processing is widespread across mouse visual areas V1, LM, and RL}

The overall high disparity selectivity found across mouse visual areas V1, LM, and RL, which harbor the largest, continuous representation of the binocular visual field in the visual cortex, matches the widespread distribution of disparity processing throughout most of the visual cortex of carnivorans and primates. The binocular disparity signals that these neurons carry are potentially critical for depth perception, because they are 
A
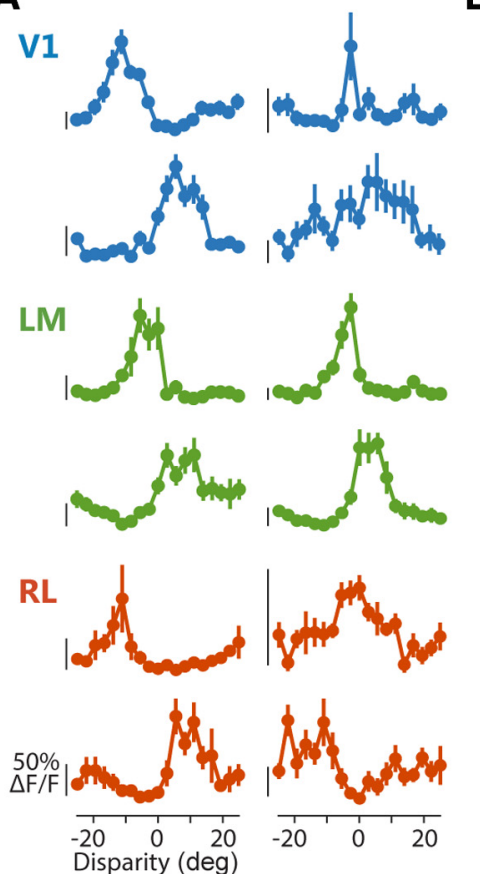

B
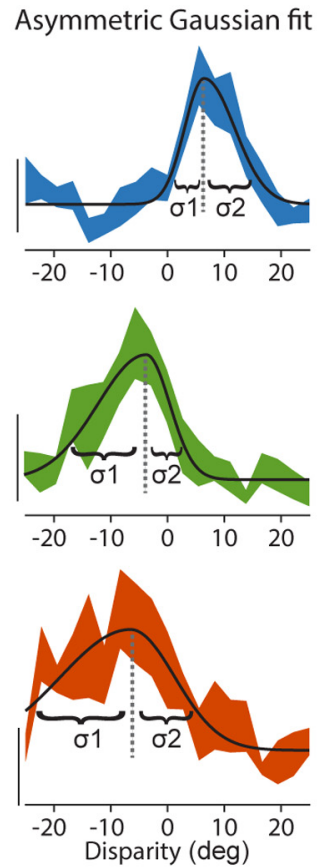

C
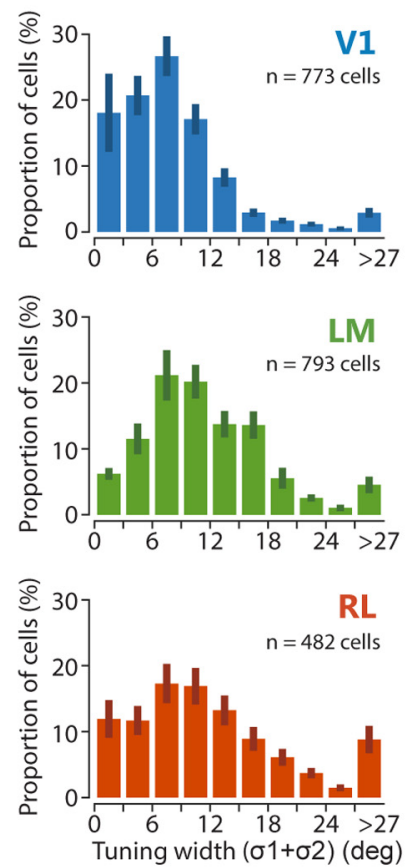

D
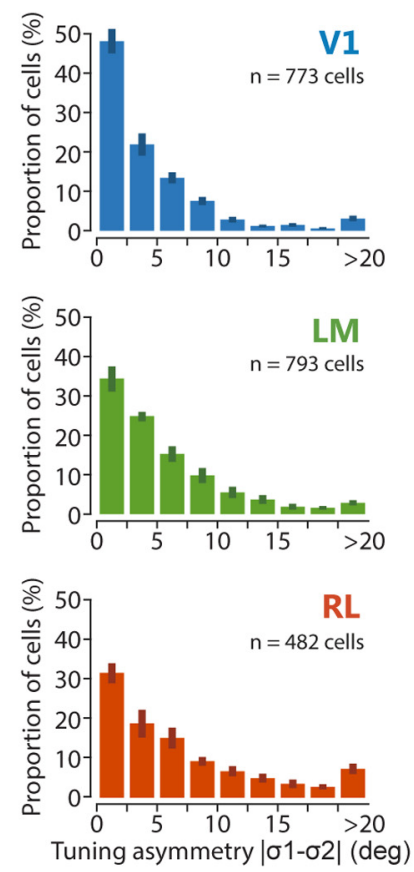

Figure 7. Characterization of disparity-tuned neurons using RDC. $\boldsymbol{A}$, Example tuning curves from different cells located in areas V1, LM, and RL as indicated by the color code. Mean fluorescence response is plotted as a function of the RDC disparities. Error bars indicate SEM $\boldsymbol{B}$, Tuning curve fit with an asymmetric Gaussian function. Three examples curve fits are shown, illustrating the tuning width parameters for the left and right sides $(\sigma 1$ and $\sigma 2)$. Scale bars in $\boldsymbol{A}, \boldsymbol{B}$ for neuronal response indicate $50 \% \Delta \mathrm{F} / \mathrm{F}$, with the bottom end of each scale bar corresponding to the baseline level $(0 \% \Delta \mathrm{F} / \mathrm{F})$. C, Distributions of disparity tuning width for each area, determined with RDC, as the sum of the two width parameters of the Gaussian fit $(\sigma 1+\sigma 2)$. $\boldsymbol{D}$, Distributions of disparity tuning asymmetry for each area, determined with cRDC as the difference between the two width parameters of the Gaussian fit (| $\sigma 1-\sigma 2 \mid)$.

essential for the construction of stereopsis by the visual system. It is important to note that some of the binocular responses we have observed might serve aspects of binocular vision other than stereopsis, like optic flow (Nityananda and Read, 2017). While neurons tuned to RDC most likely contribute to stereopsis, responses to grating stimuli need to be interpreted with more caution. Because of the circular nature of grating stimuli, in conjunction with presentation via a haploscope that did not allow optimal control of the eyes' optical axes, it cannot be ruled out that some of these neurons were driven by components of the grating stimulus that are unrelated to binocular disparity. For a complete understanding of the functional significance of disparity signals for depth processing in the mouse, it will also be valuable to compare responses to horizontal and vertical disparities, for grating stimuli, as well as for $\mathrm{CRDC}$ and aRDC. While neurons in primate visual cortex are mostly selective to horizontal rather than vertical disparities, several studies have demonstrated some contribution of vertical disparity to eye movements and depth perception (Cumming, 2002; Read, 2010). Considering the differences in eye movements (Wallace et al., 2013; Samonds et al., 2018, 2019; Choi and Priebe, 2020) and stereo-geometry of the visual system (Priebe and McGee, 2014) between primates and rodents, the processing of vertical disparity and its role in mouse vision might be quite different compared with primates. In any case, the abundance of horizontal disparity signals found in all three visual areas, especially in response to RDC, strongly suggests that mice do use binocular disparity as a depth cue to estimate object distances. While it is not fully understood how rodents move their two eyes to perceive the environment and enable binocular vision (Meyer et al., 2018, 2020; Michaiel et al., 2020), mice are capable of stereoscopic depth perception, sharing at least some of the fundamental characteristics of stereopsis with carnivorans and primates (Samonds et al., 2019).
Studies in these species have long sought to identify a cortical region specifically dedicated to stereoscopic depth processing, but failed in achieving this goal (Parker, 2007). For other visual object features, specific cortical regions have been shown to be particularly relevant. For example, primate areas V4 and MT are considered crucial centers for color and motion perception, respectively (Lueck et al., 1989; Born and Bradley, 2005). Why is binocular disparity processing so widespread across multiple areas? One possibility is that disparity processing relies on the concomitant recruitment of several areas. Similar disparity signals generated in these areas might then be differentially combined with information about other aspects of visual stimuli, such as motion, contrast, and shape, or with information deriving from other sensory modalities, to construct the percept of a $3 \mathrm{D}$ object. Another possibility is that different areas do form specialized representations of binocular disparity, thereby playing distinct disparity-related roles in constructing a 3D percept (Roe et al., 2007), such as encoding near or far space (Nasr and Tootell, 2018; La Chioma et al., 2019), supporting reaching movements, or computing visual object motion across depth (Czuba et al., 2014; Sanada and DeAngelis, 2014).

\section{Areal differences in disparity tuning curve shapes}

From V1 to extrastriate areas of primates, disparity tuning shows a progression toward broader tuning curves and increased tuning asymmetry (Cumming and DeAngelis, 2001; Prince et al., 2002b; DeAngelis and Uka, 2003). This suggests that the neuronal responses of extrastriate areas are generated by combining the disparity signals originating in V1 through specific circuits. Alternatively, some of the extrastriate responses might be generated de novo, independently of V1 (Cumming and DeAngelis, 2001; DeAngelis and Uka, 2003; Tanabe and Cumming, 2008). The asymmetry of disparity tuning curves has been related to 
A

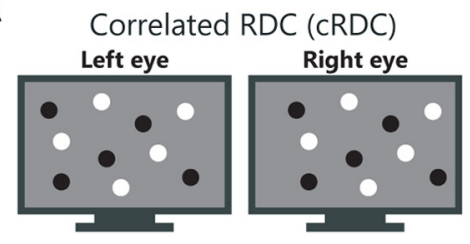

Anticorrelated RDC (aRDC)

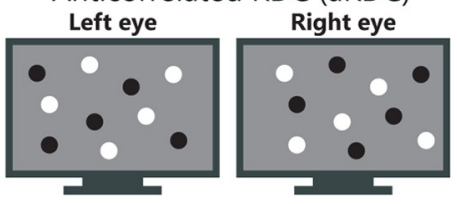

C

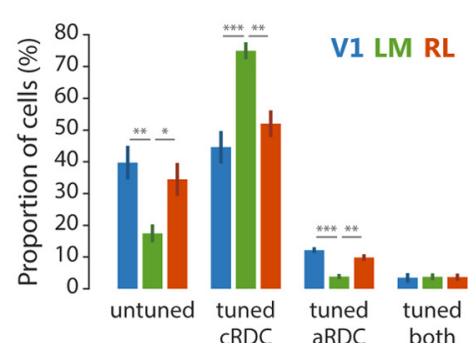

$\mathbf{E}$

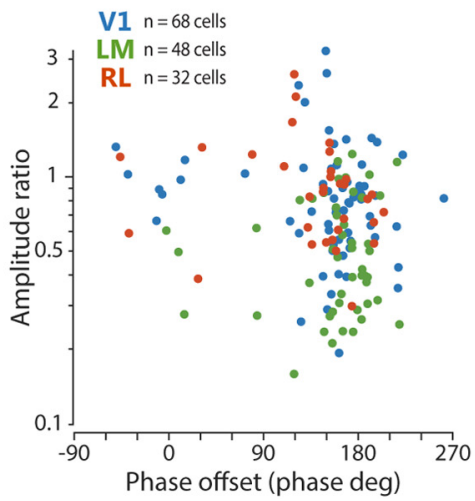

B

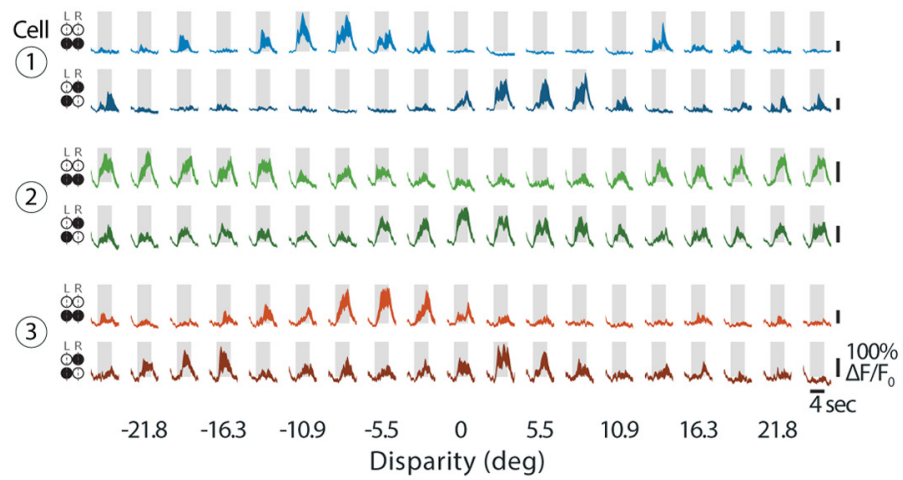

D
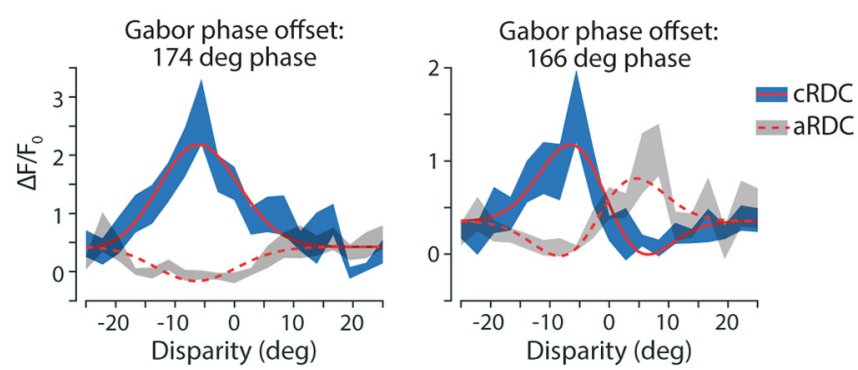

$\mathbf{F}$
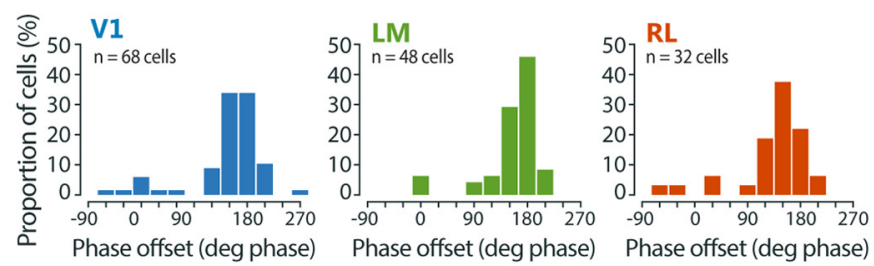

G
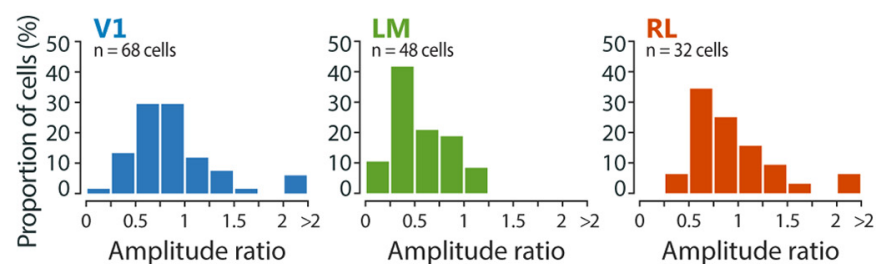

Figure 8. Area-specific responses to aRDC. $\boldsymbol{A}$, Schematic of $\mathrm{CRDC}$ and aRDC stimuli. $\boldsymbol{B}$, Visually evoked calcium traces $\left(\Delta \mathrm{F} / \mathrm{F}_{0}\right)$ of three example neurons, one from each area, in response to CRDC (upper traces, lighter shading) and aRDC (lower traces, darker shading). Fluorescence time courses are plotted as mean $\triangle \mathrm{F} / \mathrm{F}_{0} \pm \mathrm{SEM}$ (shaded areas) calculated across stimulus trials (10 repeats). Gray boxes, duration of stimulus presentation $(4 \mathrm{~s})$, bottom edge indicates baseline level $\left(0 \% \Delta \mathrm{F} / \mathrm{F}_{0}\right)$. $\mathrm{C}$, Percentage of disparity-tuned and untuned neurons, mean $\pm \mathrm{SEM}$ across imaging planes. Statistical tests: Kruskal-Wallis test across areas for each tuning group separately, followed by Bonferroni-corrected Mann-Whitney tests, with asterisks denoting significance values of post hoc tests. $\boldsymbol{D}$, Tuning curve fit with Gabor functions for two example neurons in response to CRDC (solid lines) and aRDC (dashed lines). Note tuning inversion between cRDC and aRDC, as indicated by a Gabor phase offset of around $\pm 180^{\circ}$ phase. $E$, Gabor amplitude ratio plotted against Gabor phase offset between $C R D C$ and aRDC tuning curves, for individual neurons tuned to both stimuli (V1, $n=68$ cells, 9 planes, 4 mice; LM, $n=48$ cells, 8 planes, 3 mice; RL, $n=32$ cells, 9 planes, 4 mice). $\boldsymbol{F}$, Distributions of Gabor phase offset between cRDC and aRDC tuning curves for each area. $\mathbf{G}$, Distributions of Gabor amplitude ratio between CRDC and aRDC tuning curves for each area.

two possible mechanisms put forward to explain the disparity sensitivity of cells in the visual cortex (Qian, 1997; Cumming and DeAngelis, 2001; Tsao et al., 2003). (1) In the position-shift model, the disparity tuning of a neuron arises from a spatial offset in the RF position between left and right eye, with the RF of each eye having the same spatial arrangement of subfields. (2) Alternatively, the phase-shift model proposes that a disparitytuned neuron has left and right eye RFs in the same retinal position, but with different subfield structures. The position-shift model produces symmetric disparity tuning curves, whereas the phase-shift model is expected to cause asymmetric (or odd-symmetric) tuning curves. In the mouse, we found that the disparity tuning curves of neurons in higher visual areas LM and RL have broader and more asymmetric tuning curves, in line with what has been observed in primates. The more frequent occurrence of asymmetric disparity tuning curves in higher visual areas compared with V1 suggests that the disparity sensitivity of neurons in mouse and primate visual cortex, progressing from V1 to higher visual areas, is increasingly generated by a phase-shift mechanism over a position-shift mechanism. Future work should take advantage of the experimental tractability of the mouse to investigate the neural circuitry underlying disparity tuning.

\section{Responses to binocular correlation and anticorrelation} across visual cortex

Generating coherent stereo depth perception requires the visual system to extract binocular disparity from the visual inputs to both eyes and match the image elements in one eye to the corresponding elements in the other eye. With aRDC, i.e., image 
elements having opposite contrast between the two eyes, the neurons' RFs are presented with local matches that do not correspond to globally coherent matches. Human observers, indeed, perceive stereo depth with cRDC, but generally do not with aRDC (Julesz, 1971; Cogan et al., 1993, 1995; Cumming et al., 1998; Zhaoping and Ackermann, 2018). In primates, V1 neurons maintain disparity selectivity to aRDC despite an inversion of the tuning profile (Cumming and Parker, 1997). This indicates that V1 neurons perform a low-level analysis of disparity signals and that further disambiguation in downstream areas is necessary to discard false matches and generate stereo depth perception (Parker, 2007). Areas MT and MST in the primate dorsal stream show anticorrelated responses as strong as in V1 (Takemura et al., 2001; Krug et al., 2004). In contrast, in the ventral stream, response to binocular anticorrelation is reduced in area V4 (Tanabe et al., 2004) and is abolished further down the ventral pathway in the inferior temporal cortex (Janssen et al., 2003). These findings indicate that the ventral stream solves "the correspondence problem" by performing a higher-level analysis of disparity signals, which corresponds more closely to the perception of stereo depth (for review, see Verhoef et al., 2016). Together, these studies have helped establishing an areal hierarchy for depth processing in the primate visual system.

Accumulating evidence supports the view that also the rodent visual cortex is organized into two subnetworks, which may share similarities with the ventral and dorsal streams of primates. According to anatomic classifications based on corticocortical connectivity, higher visual areas appear to be subdivided into two distinct groups (Wang et al., 2011, 2012). In one group, areas LM, LI, POR, and P, located lateral, i.e., ventral, to V1, are more densely interconnected and preferentially target ventral regions of the cortex. In the other, dorsal group, areas $\mathrm{AL}, \mathrm{RL}, \mathrm{A}, \mathrm{AM}$, and $\mathrm{PM}$, are located primarily anterior and medial to $\mathrm{V} 1$, and provide input predominantly to dorsal regions of the cortex (Wang et al., 2011, 2012). While supported by anatomic data, the existence of distinct processing streams in rodents is still quite speculative, as functional evidence to support this concept is presently scant compared with primates, and is mostly based on differences in tuning for select RF properties, like spatial and temporal frequency, orientation and motion direction (Andermann et al., 2011; Marshel et al., 2011; Roth et al., 2012; Tohmi et al., 2014; Murakami et al., 2017; Smith et al., 2017; for review, see Laramée and Boire, 2014; Glickfeld and Olsen, 2017).

In the present study, we found that the sensitivity to anticorrelated stimuli was similarly strong in areas V1 and RL, indicating that false matches are not fully rejected at this stage of disparity processing. In this respect, area RL, considered to be part of a putative dorsal subnetwork of mouse visual cortex, resembles areas MT and MST in the primate dorsal stream. In contrast, area LM showed a higher proportion of neurons tuned only to CRDC, while there were significantly fewer neurons tuned to aRDC, and their responses were weaker compared with V1 and RL. These findings suggest that area LM, which several studies consider part of a putative ventral subnetwork of mouse visual cortex, performs a higher-level analysis of disparity signals, reminiscent of V4 and inferior temporal cortex in the primate ventral stream.

In summary, our results show that sensitivity for binocular disparities is widespread across areas of mouse visual cortex, supporting the idea that mice might use this cue for depth perception (Samonds et al., 2019). Our findings also demonstrate areal specializations for disparity processing (La Chioma et al., 2019) that support a subdivision of mouse visual cortex into ventral and dorsal processing streams, which may share features with those in primates.

\section{References}

Aihara S, Yoshida T, Hashimoto T, Ohki K (2017) Color representation is retinotopically biased but locally intermingled in mouse V1. Front Neural Circuits 11:22.

Andermann ML, Kerlin AM, Roumis DK, Glickfeld LL, Reid RC (2011) Functional specialization of mouse higher visual cortical areas. Neuron 72:1025-1039.

Averbeck BB, Latham PE, Pouget A (2006) Neural correlations, population coding and computation. Nat Rev Neurosci 7:358-366.

Berens P (2009) CircStat: a MATLAB toolbox for circular statistics. J Stat Softw 31:1-21.

Born RT, Bradley DC (2005) Structure and function of visual area MT. Annu Rev Neurosci 28:157-189.

Brainard DH (1997) The psychophysics toolbox. Spat Vis 10:433-436.

Burge J, Geisler WS (2014) Optimal disparity estimation in natural stereo images. J Vis 14:1-18.

Chen TW, Wardill TJ, Sun Y, Pulver SR, Renninger SL, Baohan A, Schreiter ER, Kerr RA, Orger MB, Jayaraman V, Looger LL, Svoboda K, Kim DS (2013) Ultrasensitive fluorescent proteins for imaging neuronal activity. Nature 499:295-300.

Choi V, Priebe N (2020) Interocular velocity cues elicit vergence eye movements in mice. J Neurophysiol 124:623-633.

Cléry J, Guipponi O, Odouard S, Wardak C, Ben Hamed S (2018) Cortical networks for encoding near and far space in the non-human primate. Neuroimage 176:164-178.

Cogan AI, Lomakin AJ, Rossi AF (1993) Depth in anticorrelated stereograms: effects of spatial density and interocular delay. Vision Res 33:1959-1975.

Cogan AI, Kontsevich LL, Lomakin AJ, Halpern DL, Blake R (1995) Binocular disparity processing with opposite-contrast stimuli. Perception 24:33-47.

Cohen MR, Kohn A (2011) Measuring and interpreting neuronal correlations. Nat Neurosci 14:811-819.

Cortes C, Vapnik V (1995) Support-vector networks. Mach Learn 20:273297.

Cossell L, Iacaruso MF, Muir DR, Houlton R, Sader EN, Ko H, Hofer SB, Mrsic-Flogel TD (2015) Functional organization of excitatory synaptic strength in primary visual cortex. Nature 518:399-403.

Cumming BG (2002) An unexpected specialization for horizontal disparity in primate primary visual cortex. Nature 418:633-636.

Cumming BG, Parker AJ (1997) Responses of primary visual cortical neurons to binocular disparity without depth perception. Nature 389:280-283.

Cumming BG, DeAngelis GC (2001) The physiology of stereopsis. Annu Rev Neurosci 24:203-238.

Cumming BG, Shapiro SE, Parker AJ (1998) Disparity detection in anticorrelated stereograms. Perception 27:1367-1377.

Czuba TB, Huk AC, Cormack LK, Kohn A (2014) Area MT encodes threedimensional motion. J Neurosci 34:15522-15533.

DeAngelis GC, Uka T (2003) Coding of horizontal disparity and velocity by MT neurons in the alert macaque. J Neurophysiol 89:1094-1111.

de Vries SEJ, Lecoq JA, Buice MA, Groblewski PA, Ocker GK, Oliver M, Feng D, Cain N, Ledochowitsch P, Millman D, Roll K, Garrett M, Keenan T, Kuan L, Mihalas S, Olsen S, Thompson C, Wakeman W, Waters J, Williams D, et al. (2020) A large-scale standardized physiological survey reveals functional organization of the mouse visual cortex. Nat Neurosci 23:138-151.

Dombeck DA, Khabbaz AN, Collman F, Adelman TL, Tank DW (2007) Imaging large-scale neural activity with cellular resolution in awake, mobile mice. Neuron 56:43-57.

Dräger UC (1975) Receptive fields of single cells and topography in mouse visual cortex. J Comp Neurol 160:269-289.

Garrett ME, Nauhaus I, Marshel JH, Callaway EM (2014) Topography and areal organization of mouse visual cortex. J Neurosci 34:12587-12600.

Ghasemi A, Zahediasl S (2012) Normality tests for statistical analysis: a guide for non-statisticians. Int J Endocrinol Metab 10:486-489.

Glickfeld LL, Olsen SR (2017) Higher-order areas of the mouse visual cortex. Annu Rev Vis Sci 3:251-273. 
Gonzalez F, Perez R (1998) Neural mechanisms underlying stereoscopic vision. Prog Neurobiol 55:191-224.

Gordon JA, Stryker MP (1996) Experience-dependent plasticity of binocular responses in the primary visual cortex of the mouse. J Neurosci 16:32743286.

Grienberger C, Konnerth A (2012) Imaging calcium in neurons. Neuron 73:862-885

Han Y, Kebschull JM, Campbell RAA, Cowan D, Imhof F, Zador AM, MrsicFlogel TD (2018) The logic of single-cell projections from visual cortex. Nature 556:51-56.

Hendel T, Mank M, Schnell B, Griesbeck O, Borst A, Reiff DF (2008) Fluorescence changes of genetic calcium indicators and OGB-1 correlated with neural activity and calcium in vivo and in vitro. J Neurosci 28:73997411.

Hinkle DA, Connor CE (2005) Quantitative characterization of disparity tuning in ventral pathway area V4. J Neurophysiol 94:2726-2737.

Janssen P, Vogels R, Liu Y, Orban GA (2003) At least at the level of inferior temporal cortex, the stereo correspondence problem is solved. Neuron 37:693-701.

Julesz B (1971) Foundations of cyclopean perception. Oxford: University of Chicago Press.

Julesz B, Kropfl W, Petrig B (1980) Large evoked potentials to dynamic random-dot correlograms and stereograms permit quick determination of stereopsis. Proc Natl Acad Sci USA 77:2348-2351.

Kalatsky VA, Stryker MP (2003) New paradigm for optical imaging: temporally encoded maps of intrinsic signal. Neuron 38:529-545.

Kato D, Baba M, Sasaki KS, Ohzawa I (2016) Effects of generalized pooling on binocular disparity selectivity of neurons in the early visual cortex. Phil Trans R Soc B 371:20150266.

Kerlin AM, Andermann ML, Berezovskii VK, Reid RC (2010) Broadly tuned response properties of diverse inhibitory neuron subtypes in mouse visual cortex. Neuron 67:858-871.

Khan AG, Poort J, Chadwick A, Blot A, Sahani M, Mrsic-Flogel TD, Hofer SB (2018) Distinct learning-induced changes in stimulus selectivity and interactions of GABAergic interneuron classes in visual cortex. Nat Neurosci 21:851-859.

Kleiner M (2010) Visual stimulus timing precision in Psychtoolbox-3: tests, pitfalls and solutions. Perception 39 ECVP Abstract Supplement:189.

Kleiner M, Brainard DH, Pelli DG, Broussard CWT, Niehorster D (2007) What's new in Psychtoolbox-3? Perception 36:S14.

Ko H, Hofer SB, Pichler B, Buchanan K. a, Sjöström PJ, Mrsic-Flogel TD (2011) Functional specificity of local synaptic connections in neocortical networks. Nature 473:87-91.

Kohn A, Coen-Cagli R, Kanitscheider I, Pouget A (2016) Correlations and neuronal population information. Annu Rev Neurosci 39:237-256.

Kondo S, Yoshida T, Ohki K (2016) Mixed functional microarchitectures for orientation selectivity in the mouse primary visual cortex. Nat Commun $7: 13210$

Krug K, Cumming BG, Parker AJ (2004) Comparing perceptual signals of single V5/MT neurons in two binocular depth tasks. J Neurophysiol 92:1586-1596.

La Chioma A, Bonhoeffer T, Hübener M (2019) Area-specific mapping of binocular disparity across mouse visual cortex. Curr Biol 29:2954-2960. e5.

Laramée M-E, Boire D (2014) Visual cortical areas of the mouse: comparison of parcellation and network structure with primates. Front Neural Circuits 8:149.

Leinweber M, Zmarz P, Buchmann P, Argast P, Hübener M, Bonhoeffer T, Keller GB (2014) Two-photon calcium imaging in mice navigating a virtual reality environment. J Vis Exp 84:e50885.

LeVay S, Voigt T (1988) Ocular dominance and disparity coding in cat visual cortex. Vis Neurosci 1:395-414.

Lueck CJ, Zeki S, Friston KJ, Deiber M-P, Cope P, Cunningham VJ, Lammertsma AA, Kennard C, Frackowiak RSJ (1989) The colour centre in the cerebral cortex of man. Nature 340:386-389.

Lütcke H, Gerhard F, Zenke F, Gerstner W, Helmchen F (2013) Inference of neuronal network spike dynamics and topology from calcium imaging data. Front Neural Circuits 7:201.

Marshel JH, Garrett ME, Nauhaus I, Callaway EM (2011) Functional specialization of seven mouse visual cortical areas. Neuron 72:1040-1054.
Maruoka H, Nakagawa N, Tsuruno S, Sakai S, Yoneda T, Hosoya T (2017) Lattice system of functionally distinct cell types in the neocortex. Science 358:610-615.

Mazurek M, Kager M, Van Hooser SD (2014) Robust quantification of orientation selectivity and direction selectivity. Front Neural Circuits 8:1-17.

Meyer AF, Poort J, O'Keefe J, Sahani M, Linden JF (2018) A head-mounted camera system integrates detailed behavioral monitoring with multichannel electrophysiology in freely moving mice. Neuron 100:46-60.e7.

Meyer AF, O’Keefe J, Poort J (2020) Two distinct types of eye-head coupling in freely moving mice. Curr Biol 30:2116-2130.e6.

Michaiel AM, Abe ETT, Niell CM (2020) Dynamics of gaze control during prey capture in freely moving mice. Elife 9:e57458.

Montijn JS, Vinck M, Pennartz CMA (2014) Population coding in mouse visual cortex: response reliability and dissociability of stimulus tuning and noise correlation. Front Comput Neurosci 8:58.

Mrsic-Flogel TD, Hofer SB, Ohki K, Reid RC, Bonhoeffer T, Hübener M (2007) Homeostatic regulation of eye-specific responses in visual cortex during ocular dominance plasticity. Neuron 54:961-972.

Murakami T, Matsui T, Ohki K (2017) Functional segregation and development of mouse higher visual areas. J Neurosci 37:9424-9437.

Nasr S, Tootell RBH (2018) Visual field biases for near and far stimuli in disparity selective columns in human visual cortex. Neuroimage 168:358365.

Niell CM (2015) Cell types, circuits, and receptive fields in the mouse visual cortex. Annu Rev Neurosci 38:413-431.

Nityananda V, Read JCA (2017) Stereopsis in animals: evolution, function and mechanisms. J Exp Biol 220:2502-2512.

Ohzawa I, Freeman RD (1986) The binocular organization of simple cells in the cat's visual cortex. J Neurophysiol 56:221-242.

Pachitariu M, Stringer C, Dipoppa M, Schröder S, Rossi LF, Dalgleish H, Carandini M, Harris KD (2016) Suite2p: beyond 10,000 neurons with standard two-photon microscopy. bioRxiv 061507. doi: https://doi.org/ $10.1101 / 061507$

Parker AJ (2007) Binocular depth perception and the cerebral cortex. Nat Rev Neurosci 8:379-391.

Priebe NJ, McGee AW (2014) Mouse vision as a gateway for understanding how experience shapes neural circuits. Front Neural Circuits 8:123.

Prince SJD, Pointon AD, Cumming BG, Parker AJ (2002a) Quantitative analysis of the responses of $\mathrm{V} 1$ neurons to horizontal disparity in dynamic random-dot stereograms. J Neurophysiol 87:191-208.

Prince SJD, Cumming BG, Parker AJ (2002b) Range and mechanism of encoding of horizontal disparity in macaque V1. J Neurophysiol 87:209221.

Qian N (1997) Binocular disparity and the perception of depth. Neuron 18:359-368.

Read JCA (2010) Vertical binocular disparity is encoded implicitly within a model neuronal population tuned to horizontal disparity and orientation. PLoS Comput Biol 6:e1000754.

Read JCA, Cumming BG (2004) Ocular dominance predicts neither strength nor class of disparity selectivity with random-dot stimuli in primate V1. J Neurophysiol 91:1271-1281.

Ringach DL, Shapley RM, Hawken MJ (2002) Orientation selectivity in macaque V1: diversity and laminar dependence. J Neurosci 22:5639-5651.

Ringach DL, Mineault PJ, Tring E, Olivas ND, Garcia-Junco-Clemente P, Trachtenberg JT (2016) Spatial clustering of tuning in mouse primary visual cortex. Nat Commun 7:12270

Roe AW, Parker AJ, Born RT, DeAngelis GC (2007) Disparity channels in early vision. J Neurosci 27:11820-11831.

Rose T, Goltstein PM, Portugues R, Griesbeck O (2014) Putting a finishing touch on GECIs. Front Mol Neurosci 7:1-15.

Rose T, Jaepel J, Hübener M, Bonhoeffer T (2016) Cell-specific restoration of stimulus preference after monocular deprivation in the visual cortex. Science 352:1319-1322.

Roth MM, Helmchen F, Kampa BM (2012) Distinct functional properties of primary and posteromedial visual area of mouse neocortex. J Neurosci 32:9716-9726.

Sakatani T, Isa T (2007) Quantitative analysis of spontaneous saccade-like rapid eye movements in C57BL/6 mice. Neurosci Res 58:324-331.

Samonds JM, Geisler WS, Priebe NJ (2018) Natural image and receptive field statistics predict saccade sizes. Nat Neurosci 21:1591-1599.

Samonds JM, Choi V, Priebe NJ (2019) Mice discriminate stereoscopic surfaces without fixating in depth. J Neurosci 39:8024-8037. 
Sanada TM, DeAngelis GC (2014) Neural representation of motion-in-depth in area MT. J Neurosci 34:15508-15521.

Scholl BK, Burge J, Priebe NJ (2013) Binocular integration and disparity selectivity in mouse primary visual cortex. J Neurophysiol 109:3013-3024.

Scholl BK, Pattadkal JJ, Dilly GA, Priebe NJ, Zemelman BV (2015) Local integration accounts for weak selectivity of mouse neocortical parvalbumin interneurons. Neuron 87:424-436.

Scholl BK, Pattadkal JJ, Rowe A, Priebe NJ (2017) Functional characterization and spatial clustering of visual cortical neurons in the predatory grasshopper mouse Onychomys arenicola. J Neurophysiol 117:910-918.

Schulz DP, Sahani M, Carandini M (2015) Five key factors determining pairwise correlations in visual cortex. J Neurophysiol 114:1022-1033.

Sit KK, Goard MJ (2020) Distributed and retinotopically asymmetric processing of coherent motion in mouse visual cortex. Nat Commun 11:3565.

Smith IT, Townsend LB, Huh R, Zhu H, Smith SL (2017) Stream-dependent development of higher visual cortical areas. Nat Neurosci 20:200-208.

Sterratt DC, Lyngholm D, Willshaw DJ, Thompson ID (2013) Standard anatomical and visual space for the mouse retina: computational reconstruction and transformation of flattened retinae with the Retistruct package. PLoS Comput Biol 9:e1002921.

Takemura A, Inoue Y, Kawano K, Quaia C, Miles FA (2001) Single-unit activity in cortical area MST associated with disparity-vergence eye movements: evidence for population coding. J Neurophysiol 85:2245-2266.

Tanabe S, Cumming BG (2008) Mechanisms underlying the transformation of disparity signals from V1 to V2 in the macaque. J Neurosci 28:1130411314.

Tanabe S, Umeda K, Fujita I (2004) Rejection of false matches for binocular correspondence in macaque visual cortical area V4. J Neurosci 24:81708180 .
Tohmi M, Meguro R, Tsukano H, Hishida R, Shibuki K (2014) The extrageniculate visual pathway generates distinct response properties in the higher visual areas of mice. Curr Biol 24:587-597.

Tsao DY, Conway BR, Livingstone MS (2003) Receptive fields of disparitytuned simple cells in macaque V1. Neuron 38:103-114.

Van den Bergh G, Zhang B, Arckens L, Chino YM (2010) Receptive-field properties of V1 and V2 neurons in mice and macaque monkeys. J Comp Neurol 518:2051-2070

Verhoef B-E, Vogels R, Janssen P (2016) Binocular depth processing in the ventral visual pathway. Phil Trans R Soc B 371:20150259.

Wallace DJ, Greenberg DS, Sawinski J, Rulla S, Notaro G, Kerr JND (2013) Rats maintain an overhead binocular field at the expense of constant fusion. Nature 498:65-69.

Wang BS, Sarnaik R, Cang J (2010) Critical period plasticity matches binocular orientation preference in the visual cortex. Neuron 65:246-256.

Wang Q, Gao E, Burkhalter A (2011) Gateways of ventral and dorsal streams in mouse visual cortex. J Neurosci 31:1905-1918.

Wang Q, Sporns O, Burkhalter A (2012) Network analysis of corticocortical connections reveals ventral and dorsal processing streams in mouse visual cortex. J Neurosci 32:4386-4399.

Welchman AE (2016) The human brain in depth: how we see in 3D. Annu Rev Vis Sci 2:345-376.

Zariwala HA, Madisen L, Ahrens KF, Bernard A, Lein ES, Jones AR, Zeng H (2011) Visual tuning properties of genetically identified layer $2 / 3$ neuronal types in the primary visual cortex of Cre-transgenic mice. Front Syst Neurosci 4:16

Zhaoping L, Ackermann J (2018) Reversed depth in anticorrelated randomdot stereograms and the central-peripheral difference in visual inference. Perception 47:531-539.

Zhuang J, Ng L, Williams D, Valley M, Li Y, Garrett M, Waters J (2017) An extended retinotopic map of mouse cortex. Elife 6:e18372. 